\title{
Contact surgeries and the transverse invariant in knot Floer homology
}

\author{
Peter Ozsváth \\ ANDRÁs I. Stipsicz \\ Department of Mathematics, Columbia University, \\ New York 10027, USA \\ Rényi Institute of Mathematics, Budapest, Hungary and \\ Department of Mathematics, Columbia University, New York 10027, USA \\ Email: petero@math.columbia.edu stipsicz@renyi.hu
}

\begin{abstract}
We study naturality properties of the transverse invariant in knot Floer homology under contact $(+1)$-surgery. This can be used as a calculational tool for the transverse invariant. As a consequence, we show that the Eliashberg-Chekanov twist knots $E_{n}$ are not transversely simple for $n$ odd and $n>3$.
\end{abstract}

AMS Classification 57M27; 57R58

Keywords Legendrian knots, transverse knots, Heegaard Floer homology

\section{Introduction}

Suppose that $T \subset(Y, \xi)$ is a null-homologous transverse knot in the closed contact 3-manifold $(Y, \xi)$. According to [12, there is an invariant $\mathfrak{T}(T)$ of the transverse isotopy class of $T$, taking values in the knot Floer homology group $\operatorname{HFK}^{-}(-Y, T)$ (introduced in [21, 27]). This invariant is defined using open book decompositions and Heegaard Floer homology. For the definition, we approximate $T$ by a coherently oriented Legendrian knot $L$ and find an appropriate open book decomposition compatible with $(Y, \xi, L)$. In the Heegaard diagram corresponding to this open book decomposition there is a distinguished intersection point, giving a generator of the chain complex $\mathrm{CFK}^{-}(-Y, L)$ for knot Floer homology. The element induces a homology class $\mathfrak{L}(Y, \xi, L) \in \operatorname{HFK}^{-}(-Y, L)$, called the Legendrian invariant of $L$. Since the Legendrian invariant remains unchanged under negative stabilization, it can be viewed as an invariant $\mathfrak{T}(Y, \xi, T)$ of the transverse knot $T$. This invariant turns out to be an effective tool for studying Legendrian and transverse knots in various contact $3-$ manifolds. The specialization $U=0$ turns $\operatorname{HFK}^{-}(-Y, L)$ into $\widehat{\operatorname{HFK}}(-Y, L)$; the image of the invariant $\mathfrak{L}(Y, \xi, L)$ (and $\mathfrak{T}(Y, \xi, T))$ under this reduction is denoted by $\widehat{\mathfrak{L}}(Y, \xi, L)$ (and $\widehat{\mathfrak{T}}(Y, \xi, T)$, resp.). 
The motivation for the Legendrian and transverse invariants comes from the construction in [19], which gives an invariant for Legendrian and transverse knots in the standard contact three-sphere, taking values in the combinatorial knot Floer homology of [13, 14]; see also [9, 24] for other constructions and [15, 30] for related computations.

In this paper we show that the invariant $\mathfrak{L}$ (and hence $\mathfrak{T}$ ) enjoys a simple transformation rule under the change of the contact 3-manifold by contact $(+1)$-surgery.

Theorem 1.1 Suppose that $L, S \subset(Y, \xi)$ are disjoint Legendrian knots in the contact 3-manifold $(Y, \xi)$, with $L$ null-homologous and oriented. Let $\left(Y_{S}, \xi_{S}\right)$ denote the contact 3-manifold we get by performing contact $(+1)$-surgery along $S$, while $L_{S}$ will denote the Legendrian knot $L$ viewed in $\left(Y_{S}, \xi_{S}\right)$. Suppose that $L_{S}$ is null-homologous in $Y_{S}$. The surgery gives rise to maps

$$
F_{S, \mathfrak{s}}: \operatorname{HFK}^{-}(-Y, L) \rightarrow \operatorname{HFK}^{-}\left(-Y_{S}, L_{S}\right),
$$

where $\mathfrak{s}$ is a $\operatorname{Spin}^{c}$ structure on the cobordism $W$ from $Y$ to $Y_{S}$. There is a unique $\mathfrak{s}$ for which

$$
F_{S, \mathfrak{s}}(\mathfrak{L}(Y, \xi, L))=\mathfrak{L}\left(Y_{S}, \xi_{S}, L_{S}\right)
$$

holds, and for all other Spinc structure $s$ the map $F_{S, s}$ is trivial on $\mathfrak{L}(Y, \xi, L)$. A similar identity holds for the Legendrian invariant $\widehat{\mathfrak{L}}$ in $\widehat{\mathrm{HFK}}$.

This has the following immediate consequence for the transverse invariant:

Corollary 1.2 Let $T \subset(Y, \xi)$ be a null-homologous transverse knot and $S \subset(Y, \xi)$ a Legendrian knot disjoint from $T$. Let $\left(Y_{S}, \xi_{S}, T_{S}\right)$ denote the result of the contact $(+1)$-surgery along $S$, and suppose that $T_{S}$ (the knot $T$ viewed in $\left.\left(Y_{S}, \xi_{S}\right)\right)$ is null-homologous in $Y_{S}$. Then there is a unique $\operatorname{Spin}^{c}$ structure $\mathfrak{s}$ for which $F_{S, \mathfrak{s}}(\mathfrak{T}(Y, \xi, T))=\mathfrak{T}\left(Y_{S}, \xi_{S}, T_{S}\right)$ and for all other Spin ${ }^{c}$ structure $s$ the map $F_{S, s}$ is trivial on $\mathfrak{T}(T)$. Similar statement holds for the invariant $\widehat{\mathfrak{T}}$ in $\widehat{\mathrm{HFK}}$.

This theorem simplifies the computation of $\mathfrak{L}$ and $\mathfrak{T}$ for many interesting cases, allowing us to use it to distinguish transversely non-isotopic transverse knots in the same knot type with the same self-linking number. Recall that a knot type is said to be transversely simple if it has no such pairs of transverse representatives. The first examples of transversely non-simple knot types were found by EtnyreHonda [7] and Birman-Menasco [3], and further examples were found in [15]. We will use Theorem 1.1 to show the following: 
Theorem 1.3 The Eliashberg-Chekanov twist knot $E_{n}$ shown in Figure 1 is not transversely simple for $n$ odd and $n>3$. In fact, for $n$ odd there are at least $\left\lceil\frac{n}{4}\right\rceil$ transverse knots in the standard contact 3 -sphere $\left(S^{3}, \xi_{s t}\right)$ with self-linking number equal to 1 , all topologically isotopic to $E_{n}$, yet not pairwise transverse isotopic.

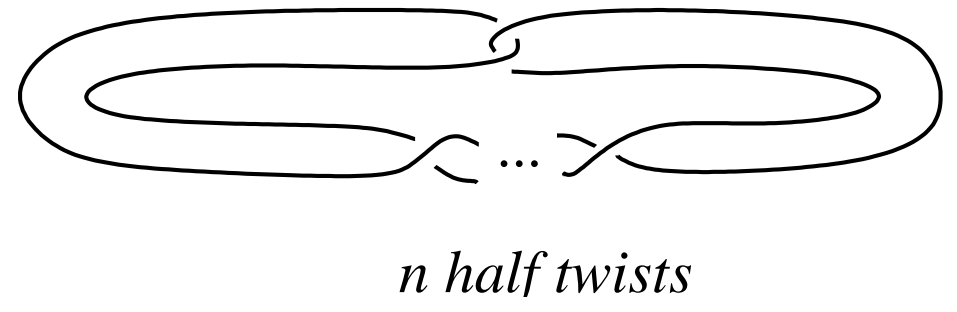

Figure 1: The Eliashberg-Chekanov knot $E_{n}$.

As a special case, we have the following:

Corollary 1.4 The twist knot which is the mirror of $7_{2}$ in Rolfsen's table is not transversely simple.

Proof The knot $m\left(7_{2}\right)$ is the twist knot $E_{5}$ from Figure1; hence Theorem 1.3 applies with $n=5$.

Remark 1.5 Recall that by defining and computing the Eliashberg-Chekanov DGA for Legendrian representatives of the $5_{2}$ knot, Chekanov [4] showed that the knot type $5_{2}$ is not Legendrian simple, that is, the knot type admits Legendrian representatives which are not Legendrian isotopic, though they do have the same classical invariants (Thurston-Bennequin and rotation numbers). The result was extended in [5] for all Eliashberg-Chekanov knots $E_{n}(n \geq 3$ and odd). Notice that the DGA's used in these proofs vanish for stabilized knots, hence generally these invariants cannot be used to distinguish Legendrian approximations of transverse knots. The question of whether or not $5_{2}$ is transversely simple remains open. 1

In fact, the same proof leads to finding further transversely non-simple twobridge knots in the standard contact 3-sphere; the precise statement is deferred

\footnotetext{
${ }^{1}$ In fact, very recently, a classification of the Legendrian and transverse isotopy classes of $E_{n}$ has been announced in [6]
} 
to Section 5. The proof of Theorem 1.3, resting on the transformation rule given by Theorem 1.1, uses two further ingredients, which we spell out next.

Suppose that $F$ is a fixed Seifert surface for $L$. The invariant $\mathfrak{L}(L) \in \operatorname{HFK}^{-}(-Y, L)$ admits an Alexander grading $A_{F}(\mathfrak{L}(L))$ and (provided the $\operatorname{Spin}^{c}$ structure of the contact structure has torsion first Chern class) a Maslov grading $M(\mathfrak{L}(L))$. The Thurston-Bennequin and rotation numbers $\operatorname{tb}(L)$ and $\operatorname{rot}_{F}(L)$ of the nullhomologous Legendrian knot $L$ can be defined in the standard way, cf. Section 4. The relationship between these numerical invariants of $\mathfrak{L}(L)$ and $L$ is given as follows:

Theorem 1.6 Let $L \subset(Y, \xi)$ be a Legendrian knot in the contact 3-manifold $(Y, \xi)$ and suppose that $F$ is a Seifert surface for $L$. Then, the chain $\mathfrak{L}(L) \in$ $\mathrm{CFK}^{-}(-Y, L)$ is supported in Alexander grading

$$
2 A_{F}(\mathfrak{L}(L))=\operatorname{tb}(L)-\operatorname{rot}_{F}(L)+1 .
$$

If $c_{1}(\xi)$ is torsion, then the Maslov grading of $\mathfrak{L}(L)$ is determined by

$$
2 A(\mathfrak{L}(L))-M(\mathfrak{L}(L))=d_{3}(\xi),
$$

where $d_{3}(\xi)$ is the 3-dimensional invariant (also known as the Hopf invariant) of the 2-plane field underlying the contact structure $\xi$.

Since the self-linking number $\mathrm{sl}_{F}(T)$ of a transverse knot $T$ can be computed from its Legendrian approximation $L$ as

$$
\operatorname{sl}_{F}(T)=\operatorname{tb}(L)-\operatorname{rot}_{F}(L),
$$

we get the following:

Corollary 1.7 For a contact 3-manifold $(Y, \xi)$ and transverse knot $T$ the transverse invariant $\mathfrak{T}(T)$ has Alexander grading $A_{F}(\mathfrak{T}(T))=\frac{1}{2}\left(\operatorname{sl}_{F}(T)+1\right)$ and (provided $c_{1}(\xi)$ is torsion) Maslov grading $M(\mathfrak{T}(T))=\operatorname{sl}(T)+1-d_{3}(\xi)$. Similar identities hold for the invariant in $\widehat{\mathrm{HFK}}$.

The second ingredient in the proof of Theorem 1.3 is a refinement of the invariant defined in [12. Recall, that $\mathfrak{L}(L)$ was only defined up to graded automorphisms of the ambient knot Floer homology group $\operatorname{HFK}^{-}(-Y, L)$. By defining the action of the mapping class group $\operatorname{MCG}(Y, L)$ of the knot complement on the knot Floer homology, we will show that the Legendrian isotopy class of $L$ gives rise to an element in $\operatorname{HFK}^{-}(-Y, L) / \pm \operatorname{MCG}(Y, L)$ rather than in its quotient $\operatorname{HFK}^{-}(-Y, L) / \operatorname{Aut}(Y, L)$ (here, by $\pm \operatorname{MCG}(Y, L)$ we are emphasizing that we divide out also by the automorphism gotten by multiplication by -1 ). 
Since $\pm \operatorname{MCG}(Y, L)$ is typically much smaller than $\operatorname{Aut}(Y, L)$, this lift enables us to use the invariant much more effectively. By using $\mathbb{F}=\mathbb{Z} / 2 \mathbb{Z}$-coefficients, the action of -1 can be ignored, and in the following we will apply this choice of coefficient groups. The precise formulation of the Heegaard Floer theoretic result showing the existence of the MCG-action will be given in Section 6 .

The paper is organized as follows. Section 2 is devoted to the collection of preliminary results, and an explanation how the invariant is lifted from $\mathrm{HFK}^{-} / \mathrm{Aut}$ to $\mathrm{HFK}^{-} / \pm \mathrm{MCG}$. The proof of Theorem 1.1 is given in Section 3 , and we verify the formulae computing the Alexander and Maslov gradings of $\mathfrak{L}$ in Section 4. We study Eliashberg-Chekanov knots - and certain further two-bridge knots - in Section 5, and in particular give the proof of Theorem 1.3. Finally in Section 6 the necessary Heegaard Floer theoretic discussion for defining the action of $\operatorname{MCG}(Y, L)$ on the knot Floer groups is given.

Acknowledgements. The authors wish to thank Paolo Ghiggini, András Juhász, Paolo Lisca, Zoltán Szabó and Dylan Thurston for interesting and helpful discussions. The authors are especially grateful to Sucharit Sarkar for some discussions of naturality. PSO was supported by NSF grant number DMS0505811 and FRG-0244663. AS acknowledges support from the Clay Mathematics Institute. AS was also partially supported by OTKA T67928 and by Marie Curie TOK project BudAlgGeo.

\section{Preliminaries}

We review some of the constructions which will be used throughout the paper.

\subsection{Knot Floer homology}

We set up notation for knot Floer homology, following [21] (see also [27]). Let $\Sigma$ be a closed, oriented surface of genus $g$, and $\alpha=\left\{\alpha_{1}, \ldots, \alpha_{g}\right\}$ be a $g-$ tuple of homologically linearly independent, pairwise disjoint circles; and let $\beta=\left\{\beta_{1}, \ldots, \beta_{g}\right\}$ be another such $g$-tuple of circles. The triple $(\Sigma, \alpha, \beta)$ is a Heegaard diagram specifying a closed, oriented 3 -manifold $Y$, built as follows. We start with the zero-handle, and then regard the $\alpha$-curves as belt circles of 1 -handles attached to this zero-handle, and the $\beta$-curves as attaching circles of 2 -handles. To complete $Y$, we attach the unique 3 -handle.

Fixing two points $z, w \in \Sigma$ in the complement of the $\alpha$-and $\beta$-curves, an oriented knot $K \subset Y$ is specified as follows. Connect $z$ to $w$ by a standardly 
embedded arc disjoint from the attaching disks in the handlebody determined by the $\alpha$-curves and $w$ to $z$ by such an arc in the handlebody of the $\beta$-curves. Notice that the definition, in fact, equips $K$ with an orientation. Consider $\operatorname{Sym}^{g}(\Sigma)$, equipped with the totally real submanifolds

$$
\mathbb{T}_{\alpha}=\alpha_{1} \times \ldots \times \alpha_{g} \text { and } \mathbb{T}_{\beta}=\beta_{1} \times \ldots \times \beta_{g} .
$$

A suitable adaptation of Lagrangian Floer homology in this context results in the knot Floer homology groups $\operatorname{HFK}^{-}(Y, K)$, which are the homology groups of a chain complex $\operatorname{CFK}^{-}(Y, K)$ defined over $\mathbb{F}[U]$, which is freely generated by intersection points $\mathbb{T}_{\alpha} \cap \mathbb{T}_{\beta}$. In the case where $Y$ is a rational homology three-sphere, these groups are bigraded,

$$
\operatorname{HFK}^{-}(Y, K)=\oplus_{d \in \mathbb{Q}, \mathbf{s} \in \operatorname{Spin}^{c}(Y, K)} \operatorname{HFK}_{d}^{-}(Y, K, \mathbf{s}),
$$

where $d$ is the Maslov grading and $\mathbf{s}$, which runs through relative Spin ${ }^{c}$ structures on $Y-K$, is the Alexander grading. In cases where $Y$ is not a rational homology sphere, we impose the assumption that $K$ is null-homologous, and we work with Heegaard diagrams satisfying suitable admissibility hypotheses as in [23. Even under these hypotheses, when $b_{1}(Y)>0$, the Maslov grading is no longer a rational number (except when we consider relative $\operatorname{Spin}^{c}$ structures whose first Chern class is torsion). Unless otherwise stated, we will work with $\mathbb{F}=\mathbb{Z} / 2 \mathbb{Z}$ coefficients.

\subsection{Legendrian invariants}

We briefly recall the construction of the Legendrian invariant from [12] (compare also [10, 24]).

For a Legendrian knot $L$ in a contact 3-manifold $(Y, \xi)$ a Heegaard decomposition adapted to this situation can be found by the following recipe. Choose an open book decomposition of $Y$ compatible with the contact structure $\xi$ in such a way that $L$ is a homologically essential curve on one of the pages of the open book. Such choice is possible, as can be easily verified either by the application of Giroux's algorithm for constructing open book decompositions for contact 3-manifolds through contact cell-decompositions, or by the algorithm of Akbulut and Ozbagci [1, cf. also [2]. The open book decomposition, in turn, provides a Heegaard decomposition, with Heegaard surface given as the union of two pages $P_{+1}$ and $P_{-1}$ of the open book, and $\alpha$ - and $\beta$-curves given by the following procedure. Choose $\operatorname{arcs} a_{i}$ for $i=1, \ldots, n$ in the page $P_{+1}$ of the open book which are disjoint and represent a basis of $H_{1}\left(P_{+1}, \partial P_{+1}\right)$; i.e., by cutting $P_{+1}$ open along the $a_{i}$ we get a disk. Let $b_{i}$ be a slight perturbation 
of $a_{i}$, chosen so that $b_{i}$ is disjoint from all $a_{j}$ with $j \neq i$, and intersects $a_{i}$ transversely in a single intersection point with orientation +1 , as pictured in Figure 2(cf. also [10, 12]). In the presence of $L$, the system $\left\{a_{i}\right\}$ can be chosen

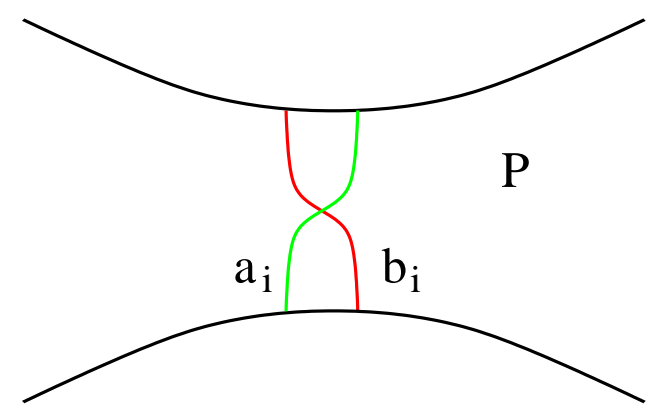

Figure 2: The $\operatorname{arcs} a_{i}$ and $b_{i}$.

in a way that only $a_{1}$ intersects $L$, and this intersection is a single transverse point. Let us take $\alpha_{i}$ to be the union of $a_{i}$ with its image under the identity map on the opposite page $P_{-1}$, while $\beta_{i}$ is the union of $b_{i}$ together with its image under the monodromy map $\phi$ (regarded as a map $\phi: P_{+1} \rightarrow P_{-1}$ ) of the open book decomposition. Clearly, $P_{+1}-a_{1}-\ldots-a_{n}-b_{1}-\ldots-b_{n}$ consists of $2 n+1$ components. For each $i=1, \ldots, n$, we have two components whose boundary consists of an arc in $a_{i}$, an arc in $b_{i}$, and an arc in $\partial P_{+1}$. There is one remaining component (whose boundary meets all the $a_{i}$ and $b_{i}$ ), and we place the basepoint $w$ in this region. Moreover, we place $z$ in one of the two remaining components meeting $a_{1}$ and $b_{1}$. We choose this component so that the induced orientation coincides with the given orientation of the Legendrian knot $L$. (Recall that we obtain an orientation on $L$ by orienting its subarc in $P_{+1}-a_{1}-\ldots-a_{n}$ so as to go from $w$ to $z$.) In this manner, we obtain a doubly-pointed Heegaard diagram $(\Sigma, \alpha, \beta, w, z)$ for the oriented Legendrian knot $L \subset Y$.

Definition 2.1 The doubly-pointed Heegaard diagram $(\Sigma, \alpha, \beta, w, z)$ constructed above is said to be adapted to the open book decomposition represented by the surface $P$, monodromy map $\phi$, and Legendrian knot $L \subset(Y, \xi)$.

Remark 2.2 Notice that $z$ and $w$ specify a smooth isotopy class of knots by connecting $z$ to $w$ on $P$ through $a_{1}$ and $w$ to $z$ through $b_{1}$. In fact, these data uniquely specify a Legendrian isotopy class of Legendrian knots, as it is shown in [12, Theorem 2.7]. 
Following the convention of [10], we reverse the roles of the $\alpha$ - and the $\beta$ circle, hence we examine the Heegaard decomposition $(\Sigma, \beta, \alpha)$. Such a change reverses the orientation of the knot, hence in order to keep the given orientation of the knot, we also switch the roles of $z$ and $w$, giving $(\Sigma, \beta, \alpha, z, w)$. With the reversal of the roles of the $\alpha$-and $\beta$-circles, the distinguished intersection point $\mathbf{x}=\left(a_{1} \cap b_{1}, \ldots, a_{n} \cap b_{n}\right) \in \mathbb{T}_{\alpha} \cap \mathbb{T}_{\beta}$ becomes be a cycle in $\operatorname{CFK}^{-}(-Y, L)$. In [12] it was shown that the homology class $\mathfrak{L}(L) \in \mathrm{HFK}^{-}(-Y, L)$ represented by $\mathbf{x}$ is independent of the choices made in its definition, i.e., from the choice of the adapted open book decomposition and the basis $\left\{a_{1}, \ldots, a_{n}\right\}$. In addition, if $L_{1}, L_{2}$ are Legendrian isotopic Legendrian knots, then there is an isomorphism $\operatorname{HFK}^{-}\left(-Y, L_{1}\right) \rightarrow \operatorname{HFK}^{-}\left(-Y, L_{2}\right)$ mapping $\mathfrak{L}\left(L_{1}\right)$ to $\mathfrak{L}\left(L_{2}\right)$. Viewing $\operatorname{HFK}^{-}(-Y, L)$ as an abstract group, the element $\mathfrak{L}\left(L_{1}\right)$ is therefore defined only up to identification of $\operatorname{HFK}^{-}\left(-Y, L_{1}\right)$ with $\operatorname{HFK}^{-}\left(-Y, L_{2}\right)$, providing the following:

Theorem 2.3 ([12]) The element $\mathfrak{L}(L)$ is an invariant of the oriented Legendrian knot $L$, with values in the graded module $\operatorname{HFK}^{-}(-Y, L)$, modulo its graded automorphisms. For a transverse knot $T$ and Legendrian approximation $L$, the class $\mathfrak{L}(L)$ (being invariant under negative stabilization) is an invariant

$$
\mathfrak{T}(T) \in \operatorname{HFK}^{-}(-Y, L) / \operatorname{Aut}\left(\operatorname{HFK}^{-}(-Y, L)\right)
$$

of the transverse isotopy class of $T$.

The above theorem supplies an invariant in knot Floer homology, modulo automorphisms. There are various strengthenings of the above statements, resulting from the types of restrictions one can naturally place on the allowed automorphisms. An example of such strengthening was given in [12 for connected sums. In a slightly different direction, on the crudest level, if $T_{1}$ and $T_{2}$ are two different transverse realizations of the same knot, and $\mathfrak{T}$ for one of them vanishes while for the other does not, then the above theorem ensures that $T_{1}$ and $T_{2}$ are not transversely isotopic. But $\mathrm{HFK}^{-}$has more algebraic structure than merely a bigraded $\mathbb{F}[U]$-module: it is naturally the homology group of an associated graded object of a filtered complex; as such, it comes equipped with higher differentials. Thus, if $T_{1}$ and $T_{2}$ both have non-vanishing transverse invariant, but $d_{1}\left(\mathfrak{T}\left(T_{1}\right)\right)$ vanishes while $d_{1}\left(\mathfrak{T}\left(T_{2}\right)\right)$ does not, then $T_{1}$ and $T_{2}$ are not transversely isotopic. This refined structure was used to distinguish transversally non-isotopic knots in the combinatorial context in [15].

Sometimes such algebraic properties are insufficient to distinguish transverse knots, and it becomes necessary to use more refined geometric tools. Below we will describe the lift of the Legendrian invariant from $\mathrm{HFK}^{-} / \mathrm{Aut}$ to $\mathrm{HFK}^{-} / \pm$ 
MCG. To this end, consider a knot $K \subset Y$ and let $\operatorname{Diff}^{+}(Y, K)$ denote the space of diffeomorphisms from $Y$ to itself which fix $K$ pointwise (or, equivalently, fix a point $p$ on $K)$. Let $\operatorname{Diff}_{0}^{+}(Y, K)$ be the set of those elements in $\operatorname{Diff}^{+}(Y, K)$ which can be connected to the identity map (through a one-parameter family of elements in $\left.\operatorname{Diff}^{+}(Y, K)\right)$. Let $\operatorname{MCG}(Y, K)$ denote the mapping class group of a knot complement, that is,

$$
\operatorname{MCG}(Y, K)=\frac{\operatorname{Diff}^{+}(Y, K)}{\operatorname{Diff}_{0}^{+}(Y, K)} .
$$

The tools of [25], adapted to the context of links, lead to an induced action of $\operatorname{MCG}(Y, K)$ on $\operatorname{HFK}^{-}(Y, K)$, which will be spelled out in Section 6. More generally, a diffeomorphism of $(Y, K, p)$ to $\left(Y^{\prime}, K^{\prime}, p^{\prime}\right)$ (where here $p \in K \subset Y$ and $p^{\prime} \in K^{\prime} \subset Y^{\prime}$ ) induces a well-defined map on $\mathrm{HFK}^{-}$. (See Theorem 6.6, and the remarks afterwards.) This concept leads us to the following refinement of Theorem 2.3.

Theorem 2.4 If $L \subset(Y, \xi)$ is a null-homologous Legendrian knot resp. $T \subset(Y, \xi)$ is a null-homologous transverse knot, then the invariant $\mathfrak{L}(L)$, resp. $\mathfrak{T}(T)$ naturally takes values in $\operatorname{HFK}^{-}(-Y, L) / \pm \operatorname{MCG}(Y, L)$; i.e. if $L_{1}$ and $L_{2}$ are Legendrian resp. transverse realizations of the same knot type $K$ whose invariants $\mathfrak{L}$ resp. $\mathfrak{T}$ lie in different orbits in $\operatorname{HFK}^{-}(-Y, K)$ under the group generated by multiplication by -1 and the mapping class group action of the knot complement, then $L_{1}$ and $L_{2}$ are not Legendrian resp. transversely isotopic.

Proof Fix a knot $L \subset Y$ in the knot type $K$, and fix a point $p \in L$, and consider $\operatorname{HFK}^{-}(-Y, L, p)$. For a Legendrian representative $L_{1}$ of $K$ and a point $p_{1} \in L_{1}$, consider an isotopy $\varphi_{t}$ between $(L, p)$ and $\left(L_{1}, p_{1}\right)$ with time-one map $\varphi_{1}$ inducing the isomorphism $\left(\varphi_{1}\right)_{*}: \operatorname{HFK}^{-}\left(-Y, L_{1}, p_{1}\right) \rightarrow \operatorname{HFK}^{-}(-Y, L, p)$ on the knot Floer homologies. Consider the image of $\mathfrak{L}\left(L_{1}\right)$ (defined up to a multiplication by $(-1))$ in $\operatorname{HFK}^{-}(-Y, L, p)$; this element will depend on the chosen isotopy $\varphi_{t}$. Another isotopy $\psi_{t}$ will give rise to another identification $\left(\psi_{1}\right)_{*}$, for which the composition $\left(\psi_{1}\right)_{*} \circ\left(\varphi_{1}\right)_{*}^{-1}$ is the action of the mapping class $\psi_{1} \circ \varphi_{1}^{-1} \in \operatorname{MCG}(-Y, L)$ on $\operatorname{HFK}^{-}(-Y, L, p)$. Therefore the element $\left(\varphi_{1}\right)_{*}\left(\mathfrak{L}\left(L_{1}\right)\right)$ is well-defined up to the action of $\pm \operatorname{MCG}(-Y, L)$.

Suppose that $\left(L_{2}, p_{2}\right)$ is another Legendrian knot with the property that $L_{1}$ and $L_{2}$ are Legendrian isotopic, and let $\zeta_{t}$ be the Legendrian isotopy between $L_{1}$ and $L_{2}$. Note that for any Legendrian knot $L_{0}$ and $p, p^{\prime} \in L_{0},\left(L_{0}, p\right)$ and $\left(L_{0}, p^{\prime}\right)$ are Legendrian isotopic (as can be verified using contact Hamiltonian 
functions). Thus, we can assume that the time-one map $\zeta_{1}$ carries $p_{1}$ to $p_{2}$. Therefore, we have an induced map $\left(\zeta_{1}\right)_{*}: \operatorname{HFK}^{-}\left(-Y, L_{1}\right) \rightarrow \operatorname{HFK}^{-}\left(-Y, L_{2}\right)$, which by [12, Corollary 3.6] maps $\mathfrak{L}\left(L_{1}\right)$ to $\mathfrak{L}\left(L_{2}\right)$. Hence the composition of the isotopies shows that the $\pm \mathrm{MCG}(-Y, L)$-orbit of the image of $\mathfrak{L}\left(L_{1}\right)$ is equal to the $\pm \mathrm{MCG}(-Y, L)$-orbit of the image of $\mathfrak{L}\left(L_{2}\right)$, concluding the proof. When using $\mathbb{Z} / 2 \mathbb{Z}$-coefficients, multiplication by $(-1)$ induces the trivial action, hence $\mathfrak{L}(L)$ is defined as an $\operatorname{MCG}(Y, L)$-orbit in this case.

In the following (in order to keep the discussion simpler) we will still refer to $\mathfrak{L}$ as an element of the knot Floer homology $\mathrm{HFK}^{-}$, although the particular element is just a representative of the corresponding $\pm \mathrm{MCG}$-orbit. Some of the basic properties of $\mathfrak{L}$ from [12] are summarized in the following:

Theorem 2.5 ([12]) If the contact invariant $c(Y, \xi)$ is nonzero, then any Legendrian knot $L \subset(Y, \xi)$ has nonvanishing $\mathfrak{L}$-invariants. If $c(Y, \xi)=0$ then $\mathfrak{L}(L)$ is a $U$-torsion class. If $(Y, \xi)$ is overtwisted and $L$ is a loose knot (that is, $Y-L$ is overtwisted) then $\mathfrak{L}(L)=0$.

In fact, the nonvanishing result was shown by applying the map

$$
\operatorname{HFK}^{-}(-Y, K, \mathbf{s}) \rightarrow \widehat{\mathrm{HF}}(-Y, \mathbf{s})
$$

given by the specialization $U=1$, which maps the Legendrian invariant $\mathfrak{L}(L)$ to the contact invariant $c(Y, \xi)$ of the contact structure $(Y, \xi)$ of [24]. In [12] a number of explicit computations for $\mathfrak{L}(L)$ were given by choosing the appropriate open book decompositions, and determining the homology class of the intersection point $\mathbf{x}$ from a direct analysis of the chain complex. In the next section we will show another way of computing $\mathfrak{L}$, which now will rely on a transformation rule developed for contact $(+1)$-surgeries. In this argument we will need to understand how knot Floer homology behaves under a map associated to a surgery.

\subsection{Maps induced by surgery}

Suppose that $Y$ is a three-manifold equipped with a framed knot $C$. Let $Y_{f}(C)$ denote the three-manifold obtained as surgery with the prescribed framing $f$ along $C$ in $Y$. The triple $(Y, C, f)$ can be described by a Heegaard triple $(\Sigma, \alpha, \beta, \gamma)$, where $(\Sigma, \alpha, \beta)$ gives $Y$ and $(\Sigma, \alpha, \gamma)$ gives $Y_{f}(C)$. By counting holomorphic triangles in $\operatorname{Sym}^{g}(\Sigma)$ with boundaries on the totally real tori 
$\mathbb{T}_{\alpha}, \mathbb{T}_{\beta}$ and $\mathbb{T}_{\gamma}$, and choosing a particular cycle in the chain complex of $(\Sigma, \beta, \gamma)$, we get a map

$$
\widehat{F}_{C}: \widehat{\mathrm{HF}}(Y) \rightarrow \widehat{\mathrm{HF}}\left(Y_{f}(C)\right)
$$

(It is shown in 25] that the map $\widehat{F}_{C}$ does not depend on the particular choices and, in fact, is an invariant of the 4-dimensional surgery cobordism.)

If $\mu$ denotes a meridian for $C$, then we can think of $f+\mu$ as a new framing. In fact, $Y_{f+\mu}(C)$ can be regarded as surgery along a framed knot $\left(C^{\prime}, f^{\prime}\right) \subset Y_{f}(C)$ and $Y$ can be regarded as the result of a surgery along $\left(C^{\prime \prime}, f^{\prime \prime}\right) \subset Y_{f+\mu}(C)$. If $Y_{1}, Y_{2}$, and $Y_{3}$ are three three-manifolds which are related in this manner, i.e. $Y_{1}=Y, Y_{2}=Y_{f}(C)$, and $Y_{3}=Y_{f+\mu}(C)$, then we say that the cyclically ordered triple $\left(Y_{1}, Y_{2}, Y_{3}\right)$ forms a distinguished triangle. Note that the roles of $Y, Y_{f}(C)$, and $Y_{f+\mu}(C)$ are cyclically symmetric. In fact, all three threemanifolds are obtained by Dehn filling the same three-manifold $M$ with torus boundary along three different surgery slopes, which meet pairwise in a single point. According to [22], the maps $\widehat{F}_{C}, \widehat{F}_{C^{\prime}}$ and $\widehat{F}_{C^{\prime \prime}}$ fit into an exact triangle.

This construction can be refined to the case of knot Floer homology, as in [21]. Specifically, suppose that $K \subset Y-C$ is a null-homologous knot. In this case, $K$ naturally induces a null-homologous knot $K^{\prime}$ inside $Y_{f}(C)$, and $C$ gives rise to the map

$$
\widehat{F}_{C}: \widehat{\operatorname{HFK}}(Y, K) \longrightarrow \widehat{\operatorname{HFK}}\left(Y_{f}(C), K^{\prime}\right)
$$

on knot Floer homology groups. Since the assumption of $K \subset Y-C$ being null-homologous implies that the linking number of $K$ with $C$ is trivial, the induced map also preserves Alexander grading, see [21, Proposition 8.1].

Definition 2.6 Suppose that $C \subset Y$ is a framed knot and $K \subset Y-C$ is a nullhomologous knot (i.e. one whose linking number with $C$ is trivial). $K$ can be thought of as a knot in $Y$, in $Y_{f}(C)$ (denoted by $K^{\prime}$ ), or in $Y_{f+\mu}(C)$ (denoted by $\left.K^{\prime \prime}\right)$. We call the triple of knots $\left\{(Y, K),\left(Y_{f}(C), K^{\prime}\right),\left(Y_{f+\mu}(C), K^{\prime \prime}\right)\right\}$ a distinguished triangle of knots.

Note once again that the roles of the three knots in a distinguished triangle are cyclically symmetric. The surgery exact triangle of [22] then has the following extension:

Theorem 2.7 [21, Theorem 8.2] If $\left\{\left(Y_{1}, K_{1}\right),\left(Y_{2}, K_{2}\right),\left(Y_{3}, K_{3}\right)\right\}$ is a distinguished triange of knots, then the corresponding Alexander grading preserving maps fit into an exact triangle 


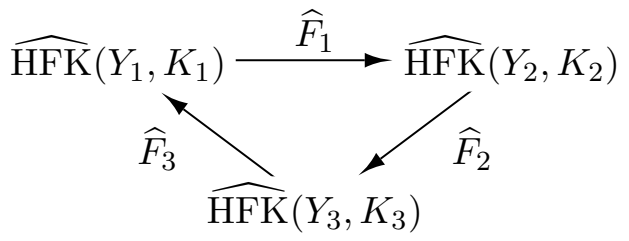

Remark 2.8 Notice that the independence of the maps $\widehat{F}_{i}$ on the chosen Heegaard triple is not claimed above - although it is plausible to expect that these maps will depend only on the 4-dimensional cobordism defined by the surgery, cf. also [29]. In our arguments we will use only the construction of these maps (given by counting holomorphic triangles) and the exactness of the triangle associated to a distinguished triangle.

The map $\widehat{F}_{C}$ on $\widehat{\mathrm{HFK}}$ defined by counting pseudo-holomorphic triangles can be extended to the case of $\mathrm{HFK}^{-}$as well, to define a map

$$
F_{C, \mathfrak{s}}: \operatorname{HFK}^{-}(Y) \rightarrow \operatorname{HFK}^{-}\left(Y_{f}(C)\right) \text {. }
$$

Just as in the case for closed three-manifolds, in order for this map to be welldefined, we must fix a $\operatorname{Spin}^{c}$-equivalence class of pseudo-holomorphic triangle on the surgery cobordism. For the case of $\widehat{\mathrm{HFK}}$, this choice can be omitted, with the understanding that $\widehat{F}_{C}$ is obtained as a sum over all such choices. In the case of $\widehat{\mathrm{HFK}}$ this is allowed since the map on $\widehat{\mathrm{CF}}$ is trivial for all but finitely many $\operatorname{Spin}^{c}$-equivalence classes of maps (compare [25. Theorem 3.3]); but again just as in the closed case, this is no longer the case for $\mathrm{HFK}^{-}$, and a choice of a $\operatorname{Spin}^{c}$ structure $\mathfrak{s}$ on the 4 -dimensional surgery cobordism is necessary.

\section{The effect of contact $(+1)$-surgery on the Legen- drian invariant}

Consider now a null-homologous Legendrian knot $L \subset(Y, \xi)$ and another Legendrian knot $S \subset(Y, \xi)$ (disjoint from $L$ ), and perform Legendrian $(+1)$ surgery along $S$. The resulting contact 3 -manifold $\left(Y_{S}, \xi_{S}\right)$ obviously contains $L$ as a Legendrian knot, denoted by $L_{S}$; suppose that $L_{S}$ is still nullhomologous in $Y_{S}$. (This condition holds, for example, if the linking number of $L$ and $S$ is zero.) Let us choose an open book decomposition which is adapted to $(Y, \xi, S, L)$ as in Definition 2.1 that is, the open book decomposition is compatible with the contact structure $\xi$ and contains $S, L$ on one of its pages. We can further assume that $S$ and $L$ are homologically essential in the page $P$, represent different homology elements, and the complement of $S$ in $P$ is connected.

The open book decomposition gives rise to a Heegaard diagram $(\Sigma, \alpha, \beta, w, z)$ : the Heegaard surface $\Sigma$ is the union of two pages $P_{+1}$ and $P_{-1}$, which are 
oriented oppositely and the $\alpha$ - and $\beta$-curves on $P_{+1} \cup\left(-P_{-1}\right)$ are defined as $\alpha_{i}=a_{i} \cup \bar{a}_{i}$ and $\beta_{i}=b_{i} \cup \overline{\phi\left(b_{i}\right)}$, where $\phi$ represents the monodromy of the open book, $\left\{a_{i}\right\}$ is a basis in $P_{+1}$ intersecting $L$ in a unique point, and $x \mapsto \bar{x}$ represents the (orientation-reversing) identification between $P_{+1}$ and $\left(-P_{-1}\right)$. Recall that the Legendrian invariant $\mathfrak{L}(L)$ is represented by the intersection point

$$
\mathbf{x}_{0}=\prod_{i=1}^{g}\left(b_{i} \cap a_{i}\right) \in \mathbb{T}_{\beta} \cap \mathbb{T}_{\alpha},
$$

now thought of as a generator for $\mathrm{CFK}^{-}(-Y, L)$, specified by the Heegaard diagram $(\Sigma, \beta, \alpha, z, w)$.

Since $L$ and $S$ are distinct in homology, we can assume that $a_{1}$ intersects $L$ transversely in a unique point (and it is disjoint from $S$ ), $a_{2}$ intersects $S$ transversely in a unique point (and it is disjoint from $L$ ), and $a_{i}$ for $i>2$ are disjoint from both $L$ and $S$. By our choice, the open book decomposition gives a compatible open book for $\left(Y_{S}, \xi_{S}, L_{S}\right)$ as well: just compose the monodromy $\phi$ with the left-handed Dehn twist $D_{S}^{-1}$ (corresponding to the fact that we perform contact $(+1)$-surgery) along $S$. This results in a change of the Heegaard diagram for $(Y, L)$ by applying the Dehn twist $D_{S}$ to all $\phi\left(b_{i}\right)$ intersecting $S$ on $P_{-1}$. In the resulting diagram it is rather complicated to detect the effect of the handle attachment.

To simplify matters, we set up things slightly differently, as follows. Consider the doubly-pointed Heegaard diagram $(\Sigma, \alpha, \gamma, w, z)$, where here the basepoints $w$ and $z$ are placed adjacent to $a_{1}$ and $b_{1}$ as before. The curve $\gamma_{2}$ is a small perturbation of $D_{S}\left(b_{2}\right) \cup \overline{\phi\left(b_{2}\right)}$, while for $i \neq 2, \gamma_{i}$ is a small perturbation of $\beta_{i}$. Although $(\Sigma, \alpha, \gamma, w, z)$ does represent $\left(Y_{S}, L_{S}\right)$, it is not adapted to the Legendrian knot $L_{S} \subset\left(Y_{S}, \xi_{S}\right)$, in the sense of Defintion 2.1. However, if we consider the Heegaard diagram $(\Sigma, \delta, \gamma, w, z)$ where here $\delta_{i}$ are suitable small perturbations of the $\alpha_{i}$ for all $i \neq 2$, while $\delta_{2}$ is a perturbation of $D_{S}\left(a_{2}\right) \cup$ $\overline{D_{S}\left(a_{2}\right)}$, then it is easy to see that $(\Sigma, \delta, \gamma, w, z)$ is an adapted Heegaard diagram for $\left(Y_{S}, \xi_{S}, L_{S}\right)$, in the sense of Definition 2.1.

The invariant $\mathfrak{L}(L) \in \mathrm{CFK}^{-}(-Y, L)$ is represented by a cycle $\mathbf{x}_{0} \in \mathbb{T}_{\beta} \cap \mathbb{T}_{\alpha}$ for the Heegaard diagram $(\Sigma, \beta, \alpha, z, w)$; similarly, $\mathfrak{L}\left(L_{S}\right)$ is represented by the intersection point point $\mathbf{x}_{1} \in \mathbb{T}_{\gamma} \cap \mathbb{T}_{\delta}$ for the Heegaard diagram $(\Sigma, \gamma, \delta, z, w)$, thought of as an element of $\mathrm{CFK}^{-}\left(-Y_{S}, L_{S}\right)$. In order to relate the Legendrian invariants $\mathfrak{L}(L)$ and $\mathfrak{L}\left(L_{S}\right)$ we will use the intermediate diagram $(\Sigma, \gamma, \alpha, z, w)$ for $\left(-Y_{S}, L_{S}\right)$.

Specifically, we would like to find an intersection point $\mathbf{y} \in \mathbb{T}_{\gamma} \cap \mathbb{T}_{\alpha}$ representing the Legendrian invariant for knot Floer homology of $\left(-Y_{S}, L_{S}\right)$, only using the 
Heegaard diagram $(\Sigma, \gamma, \alpha, z, w)$. In this case $\gamma_{i}$ and $\alpha_{i}$ meet in a single point $x_{i}$ in $P_{+1}$ for all $i \neq 2$. Special care must be taken for $i=2$. Recall that $\gamma_{2}$ and $\alpha_{2}$ on $P_{+1}$ are a perturbation $c_{2}$ of $D_{S}\left(b_{2}\right)$, and $a_{2}$ respectively. If care is not taken, these curves will be disjoint on $P_{+1}$. However, we make a finger move on $c_{2}$ to ensure it meets $a_{2}$ in two points, as pictured in Figure 3, creating an intersection point on $P_{+1}$ representing the Legendrian invariant. Now, $\gamma_{2} \cap \alpha_{2}$ consists of two points $y_{1}$ and $y_{2}$ on $P_{+1}$. For one of these choices $y_{2}$, we have that $\mathbf{y}=\left(x_{1}, y_{2}, x_{3}, \ldots, x_{n}\right)$ is a cycle in $\mathbb{T}_{\gamma} \cap \mathbb{T}_{\alpha}$ (where the other components $x_{i}$ are $a_{i} \cap b_{i}$ as before), since there is no positive domain $D$ supported in $P_{+1}$ with $n_{z}(D)=0$ and with initial point $\mathbf{y}$. This choice is illustrated in Figure 3 ,
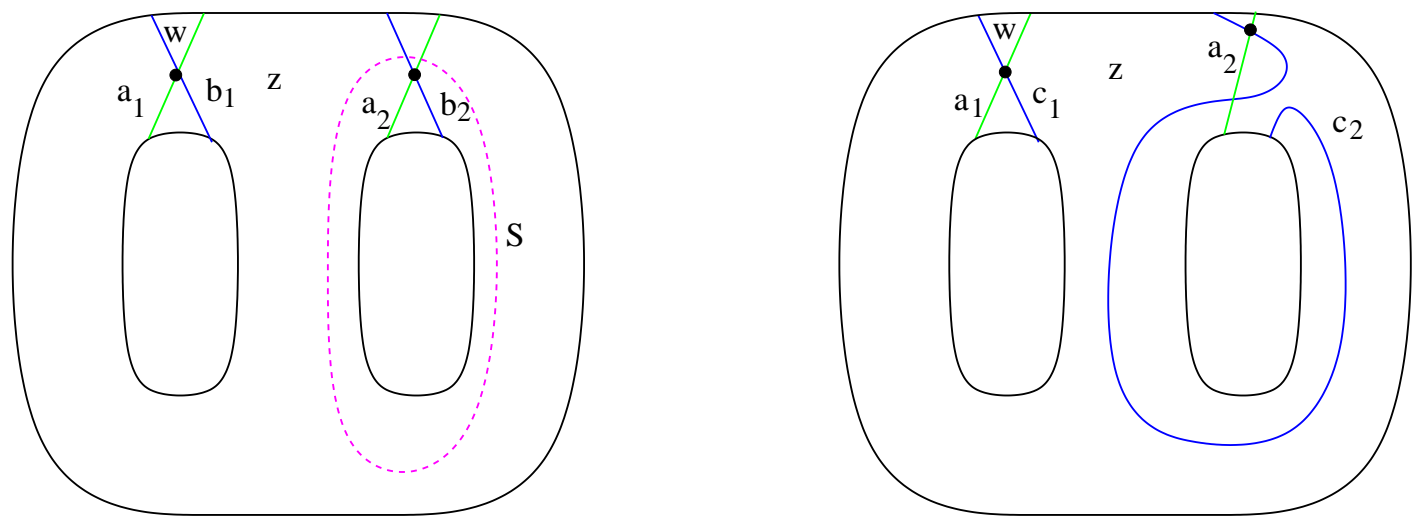

Figure 3: Intermediate cycle. We start from an open book as on the left, which supports both our initial Legendrian knot $L$, and also the Legendrian knot $S$ along which surgery is to be performed (note that this diagram is, in general, stabilized by the addition of further one-handles equipped with $a_{i}$ - and $b_{i}$-curves). $S$ here is represented by the dotted line, and $L$ is recorded by the pair of basepoints $w$ and $z$. The curve $c_{2}=D_{S}\left(b_{2}\right)$ on the right is obtained by Dehn twist along $S$ (after introducing a finger move). The components of the "intermediate intersection point" $\mathbf{y}$ are indicated by the dots on the right-hand side diagram. (Recall that the Heegaard diagram $(\Sigma, \gamma, \alpha, z, w)$ for $\left(-Y_{S}, L_{S}\right)$ is gotten by $\gamma_{i}=c_{i} \cup \overline{\phi\left(b_{i}\right)}, \alpha_{i}=a_{i} \cup \overline{a_{i}}$.)

Lemma 3.1 The intersection point $\mathbf{y} \in \mathbb{T}_{\gamma} \cap \mathbb{T}_{\beta}$ (as represented by the dia$\operatorname{gram}(\Sigma, \gamma, \alpha, z, w))$ is a cycle in $\operatorname{HFK}^{-}\left(-Y_{S}, L_{S}\right)$.

Proof The statement is proved using the same mechanism which guarantees that the distinguished intersection point for an adapted Heegaard diagram is a cycle, cf. [12, 10]. Specifically, we argue that there is no nontrivial class 
$\phi \in \pi_{2}\left(\mathbf{y}, \mathbf{y}^{\prime}\right)$ with $n_{z}(\phi)=0$. This follows from a direct analysis of the Heegaard diagram.

Recall now that

$$
F_{S, \mathfrak{s}}: \mathrm{CFK}^{-}(-Y, L) \longrightarrow \mathrm{CFK}^{-}\left(-Y_{S}, L_{S}\right)
$$

is the map induced by the map

$$
\mathrm{CFK}^{-}(\Sigma, \gamma, \beta, z, w) \otimes \mathrm{CFK}^{-}(\Sigma, \beta, \alpha, z, w) \longrightarrow \mathrm{CFK}^{-}(\Sigma, \gamma, \alpha, z, w),
$$

which is obtained by counting holomorphic triangles representing the $\operatorname{Spin}^{c}$ structure $\mathfrak{s}$, after pairing with the canonical top-dimensional homology class $\Theta$ for $\mathrm{CFK}^{-}(\Sigma, \gamma, \beta, z, w)$. (We can think of $\Theta$ as represented by some intersection point between $\mathbb{T}_{\beta}$ and $\mathbb{T}_{\gamma}$.)

Lemma 3.2 There is a unique $\operatorname{Spin}^{c}$ structure $\mathfrak{s}$ on the cobordism from $Y$ to $Y_{S}$ for which the induced map $F_{S, \mathfrak{s}}\left(\mathbf{x}_{0}\right)$ is nontrivial, where here $\mathbf{x}_{0}=\mathbf{x}(Y, \xi, L)$ represents $\mathfrak{L}(Y, \xi, L)$. For that choice, we have that

$$
F_{S, \mathfrak{s}}\left(\mathbf{x}_{0}\right)=\mathbf{y}=\left(x_{1}, y_{2}, x_{3}, \ldots, x_{n}\right) .
$$

Proof It is easy to see that the top-dimensional homology class of

$$
\operatorname{HF}^{-}\left(\#^{g-1}\left(S^{1} \times S^{2}\right)\right)=\operatorname{HFK}^{-}(\Sigma, \gamma, \beta, z, w)
$$

is represented by an intersection point $\mathbb{T}_{\gamma} \cap \mathbb{T}_{\alpha}$ supported on the page $P_{+1}$, which we denote by $\Theta_{\gamma \alpha}$. Moreover, there is a plainly visible Whitney triangle $\psi_{0} \in \pi_{2}\left(\Theta_{\gamma \alpha}, \mathbf{x}_{0}, \mathbf{y}\right)$, as illustrated on the left-hand picture in Figure 4. By the Riemann mapping theorem, this triangle has a unique pseudo-holomorphic representative. Let $\mathfrak{s}$ denote the $\operatorname{Spin}^{c}$ structure induced by this pseudoholomorphic triangle.

We claim that if $\psi \in \pi_{2}\left(\Theta_{\gamma \alpha}, \mathbf{x}_{0}, \mathbf{x}_{2}\right)$ is any homotopy class of Whitney triangles with positive underlying domain and $n_{z}(\psi)=0$, then $\mathbf{x}_{2}=\mathbf{y}$ and $\psi=\psi_{0}$. To see this, we argue that any such $\psi$ has the form $\psi_{0} * \phi$ for some $\phi \in \pi_{2}\left(\mathbf{x}_{2}, \mathbf{y}\right)$ with $n_{z}(\phi)=0$. As in the proof of Lemma 3.1, $\phi$ must be either trivial (the case where $\psi=\psi_{0}$ ) or it must have a negative local multiplicity somewhere. In the latter case, it is easy to see that $\psi_{0} * \phi$ must also have a negative local multiplicity somewhere. It now follows that $F_{S, \mathbf{t}}\left(\mathbf{x}_{0}\right)=0$ for all $\mathbf{t} \neq \mathfrak{s}$, and $F_{S, \mathfrak{s}}$ maps $\mathfrak{L}(Y, \xi, L)$ to $\mathbf{y}$, as claimed.

Next we will show that $\mathbf{y}$, in fact, represents the Legendrian invariant $\mathfrak{L}\left(Y_{S}, \xi_{S}, L_{S}\right)$. 

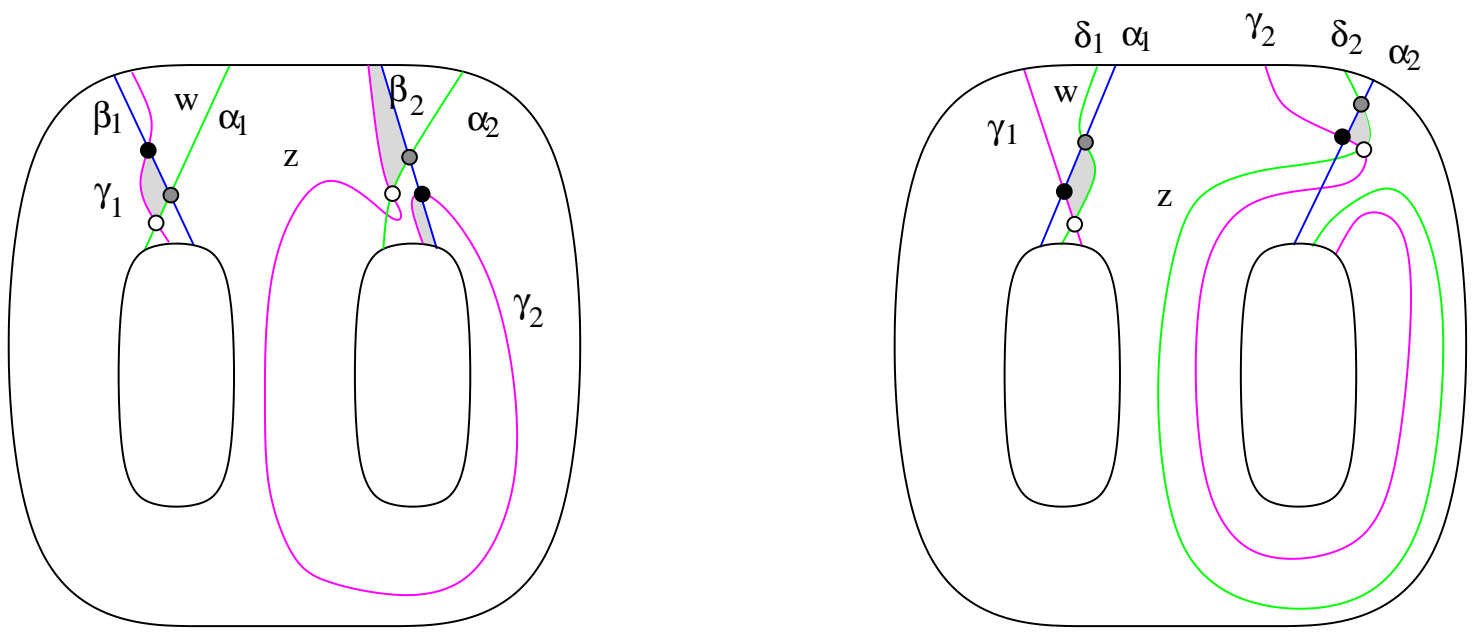

Figure 4: Intermediate triangles. On the left, we have illustrated the triangle in the Heegaard triple $(\Sigma, \gamma, \beta, \alpha, z, w)$, and on the right, we have triangles for the Heegaard triple $(\Sigma, \gamma, \alpha, \delta, z, w)$. The dark circles represent initial intersection points $\left(\Theta_{\gamma \beta} \in \mathbb{T}_{\gamma} \cap \mathbb{T}_{\beta}\right.$ on the left, $\mathbf{y} \in \mathbb{T}_{\gamma} \cap \mathbb{T}_{\alpha}$ on the right), gray circles represent intermediate ones $\left(\mathbf{x}_{0} \in \mathbb{T}_{\beta} \cap \mathbb{T}_{\alpha}\right.$ on the left, and $\Theta_{\alpha \delta} \in \mathbb{T}_{\alpha} \cap \mathbb{T}_{\delta}$ on the right), while the light ones represent the final intersection points $\left(\mathbf{y} \in \mathbb{T}_{\gamma} \cap \mathbb{T}_{\alpha}\right.$ on the left, $\mathbf{x}_{1} \in \mathbb{T}_{\gamma} \cap \mathbb{T}_{\delta}$ on the right).

Lemma 3.3 Under the homotopy equivalence

$$
H: \mathrm{CFK}^{-}(\Sigma, \gamma, \alpha, z, w) \longrightarrow \mathrm{CFK}^{-}(\Sigma, \gamma, \delta, z, w)
$$

given by handleslides, the intersection point $\mathbf{y}$ is mapped to $\mathbf{x}_{1}$, representing the Legendrian invariant of $L_{S}$.

Proof The appropriate homotopy equivalence is now induced by counting pseudo-holomorphic triangles in the Heegaard triple $(\Sigma, \beta, \gamma, \delta, z, w)$. As indicated on the right-hand picture in Figure 4, we can find an intersection point $\Theta_{\alpha \delta} \in \mathbb{T}_{\alpha} \cap \mathbb{T}_{\delta}$ which represents the top-most homology class of

$$
\mathrm{HF}^{-}\left(\#^{g}\left(S^{2} \times S^{1}\right)\right)=\operatorname{HFK}^{-}(\Sigma, \alpha, \delta, z, w) .
$$

Once again, we have a Whitney triangle, $\psi_{0} \in \pi_{2}\left(\mathbf{y}, \Theta_{\alpha \delta}, \mathbf{x}_{1}\right)$, evident on the page $P_{+1}$, also illustrated on the right-hand picture in Figure 4, where $\mathbf{x}_{1} \in$ $\mathbb{T}_{\gamma} \cap \mathbb{T}_{\delta}$ represents the Legendrian invariant. As before, if $\psi \in \pi_{2}\left(\mathbf{y}, \Theta_{\alpha \delta}, \mathbf{x}^{\prime}\right)$ is any homotopy class with positive domain and $n_{z}(\psi)=0$, then $\mathbf{x}^{\prime}=\mathbf{x}_{1}$ and $\psi=\psi_{0}$. Thus, $H(\mathbf{y})=\mathbf{x}_{1}$ as claimed. 
Proof of Theorem 1.1 According to Lemma 3.2 the map $F_{S, \mathfrak{s}}(\mathfrak{L}(L))$ is nontrivial for only one choice of $\mathfrak{s}$, for which it maps $\mathfrak{L}(L)$ to a homology class represented by $\mathbf{y}$ (which is a cycle, according to Lemma 3.1), and by Lemma 3.3 the cycle $\mathbf{y}$, in fact, represents $\mathfrak{L}\left(Y_{S}, \xi_{S}, L_{S}\right)$, concluding the proof.

In applications, it is sometimes more convenient to work with $\widehat{\mathrm{HFK}}$, the specialization of $\mathrm{HFK}^{-}$to $U=0$. Specifically, recall that the specialization $U=0$ provides a map

$$
\operatorname{HFK}^{-}(-Y, L) \rightarrow \widehat{\operatorname{HFK}}(-Y, L),
$$

and the image of the Legendrian invariant $\mathfrak{L}(L)$ under this map is denoted by $\widehat{\mathfrak{L}}(L)$. We have the corresponding maps $\widehat{F}_{S, \mathfrak{s}}$ induced by counting pseudoholomorphic triangles in the $U=0$ context. In fact, by writing

$$
\widehat{F}_{S}=\sum_{\mathfrak{s} \in \operatorname{Spin}^{c}(W)} \widehat{F}_{S, \mathfrak{s}}
$$

Theorem 1.1 has the following specialization:

Corollary 3.4 Suppose that $\left(Y_{S}, \xi_{S}, L_{S}\right)$ is the result of contact $(+1)$-surgery along $S \subset(Y, \xi, L)$ and suppose futhermore that $L \subset Y$ and $L_{S} \subset Y_{S}$ are both null-homologous Legendrian knots. Then, for the induced map $F_{S}$ on $\widehat{\operatorname{HFK}}(-Y, L)$ we have

$$
\widehat{F}_{S}(\widehat{\mathfrak{L}}(Y, \xi, L))=\widehat{\mathfrak{L}}\left(Y_{S}, \xi_{S}, L_{S}\right) .
$$

\section{Gradings}

In this section we turn our attention to the proof of Theorem 1.6, relating the bigrading of the Legendrian invariant of a Legendrian knot $L$ with the classical Legendrian invariants of $L$. We start our discussion when the ambient 3 -manifold $Y$ is an integral homology 3 -sphere, and consider the general case at the end of the subsection.

\subsection{Alexander gradings}

Suppose that $L \subset Y$ is an oriented knot in the intergral homology sphere $Y$. Let $F$ denote a Seifert surface for $L$. There is a natural map $\underline{\operatorname{Spin}^{c}}(Y, L) \longrightarrow \mathbb{Z}$ given by

$$
\mathfrak{t} \mapsto \frac{1}{2}\left\langle c_{1}(\mathfrak{t}), F\right\rangle,
$$


where a relative $\operatorname{Spin}^{c}$ structure $\mathfrak{t} \in \operatorname{Spin}^{c}(Y, L)$ is regarded as a $\operatorname{Spin}^{c}$ structure $\hat{\mathfrak{t}}$ on the 0 -surgery along $L$. The pairing $\left\langle c_{1}(\mathfrak{t}), F\right\rangle$ is interpreted as integration in the result of the 0 -surgery, i.e.

$$
\left\langle c_{1}(\mathfrak{t}), F\right\rangle=\left\langle c_{1}(\hat{\mathfrak{t}}), \hat{F}\right\rangle,
$$

where $\hat{F}$ is the surface we get by capping off the Seifert surface $F$. Since $Y$ is an integral homology sphere, the result will be independent of the particular choice of $F$.

Since any intersection point $\mathbf{x} \in \mathbb{T}_{\alpha} \cap \mathbb{T}_{\beta}$ for $(\Sigma, \beta, \alpha, z, w)$ determines a relative $\operatorname{Spin}^{c}$ structure, in view of the above definition we have an integral-valued Alexander grading belonging to each intersection point in $\mathbb{T}_{\alpha} \cap \mathbb{T}_{\beta}$.

Theorem 4.1 The integral Alexander grading of the Legendrian invariant $\mathfrak{L}(L)$ is given by the formula

$$
2 A(\mathfrak{L}(L))=\operatorname{tb}(L)-\operatorname{rot}(L)+1 .
$$

We start with some basic algebraic topology for open book decompositions, and the corresponding interpretation of the rotation number. Then, we turn to an interpretation of the rotation number and the Thurston-Bennequin invariant in terms of a compatible Heegaard diagram. This will lead to a proof of Theorem 4.1.

Recall that the open book decomposition can be given as a surface-with-boundary $P$, together with a mapping class $\phi: P \longrightarrow P$ (fixing the boundary). We can form the mapping torus, which is a three-manifold with torus boundary. Filling the tori (with appropriate slope), we form a three-manifold $Y(\phi)$ equipped with an open book decomposition. By applying positive stabilizations, we can assume that the binding $\partial P$ is connected; we will always assume this extra hypothesis on our chosen open book decomposition.

Lemma 4.2 An element $[L] \in H_{1}(P)$ is in the kernel of $H_{1}(P) \longrightarrow H_{1}(Y(\phi))$ if and only if it can be written as $[L]=\phi_{*}(Z)-Z$ for some $Z \in H_{1}(P)$.

Proof Note that $L$ has linking number zero with the binding. It then follows that if the homology class $[L]$ is in the kernel of $H_{1}(P) \longrightarrow H_{1}(Y(\phi))$, then $L$ is already null-homologous in the mapping torus $M(\phi)$ of $\phi$. Now, $M(\phi)$ is homotopy equivalent to the two-complex obtained from $P$ by attaching cylinders whose boundary components have the form $\phi_{*}(Z)-Z$. The result now follows. 
Definition 4.3 Let $f: \mathfrak{p} \longrightarrow \Sigma$ be a map of a two-manifold $\mathfrak{p}$ with boundary into a surface $\Sigma$, which has the property that the boundary of $\mathfrak{p}$ is immersed in $\Sigma$. The Euler measure of this map is defined as the relative Chern number of $f^{*}(\Sigma)$, relative to natural trivialization of its boundary inherited from the boundary, thought of as immersed curves in $\Sigma$. The Euler measure depends on $f$ only through its induced underlying two-chain. For more on the Euler measure, see [22, Section 7.1]. The Euler measure of $\mathfrak{p}$ will be denoted by $e(\mathfrak{p})$.

We descibe a way to identify the rotation number of the Legendrian knot in the Heegaard diagram. The contact distribution defines a complex line bundle over $Y(\phi)$ whose restriction to the the mapping torus (thought of as a subset of $Y(\phi))$ coincides with its "tangents along the fiber".

Lemma 4.4 Let $L \subset Y(\phi)$ be a null-homologous knot supported in a fiber $P$ for the open book decomposition. Let $\mathfrak{p}$ be a two-chain with $\partial \mathfrak{p}=[L]+(Z-$ $\left.\phi_{*}(Z)\right)$ for the one-cycle $Z$ found in Lemma 4.2. Then, the rotation number of the Legendrian knot $L$ is calculated by the Euler measure of $\mathfrak{p}$.

Proof We can construct a two-chain $F$ with boundary $L$ as follows. $F$ is composed of $\mathfrak{p}$, thought of as supported in a fiber of the open book, and then along each boundary cycle of type $Z$, we attach a cylinder which traverses the mapping torus, meeting $P$ again along the corresponding component of $\phi(Z)$. Along these cylinders, the fiberwise tangent bundle is naturally trivialized by the tangents of $F \cap P_{t}$. Along $\mathfrak{p}$, the contact bundle coincides with the tangent bundle to $\mathfrak{p}$. The result now follows.

Consider next a basis subordinate to the homologically essential knot $L \subset P$, that is, $\left\{a_{1}, \ldots, a_{g}\right\}$ is a basis for $H_{1}(P, \partial P)$ with the property that $a_{2}, \ldots, a_{g}$ are disjoint from $L$ and $a_{1}$ meets it in a single transverse intersection point. We can close off the arcs to get a basis for $H_{1}(P)$. The Thurston-Bennequin number of $L$ can be read off from these data as follows.

Lemma 4.5 Suppose $L$ is null-homologous in $Y(\phi)$ so that, according to Lemma 4.2, $[L]=\phi_{*}(Z)-Z$. Then, writing $Z$ in the above basis as

$$
Z=\sum_{i=1}^{g} n_{i} \cdot a_{i}
$$

we find that $n_{1}$ is the Thurston-Bennequin invariant of $L$, where here we have oriented $a_{1}$ so that $\# L \cap a_{1}=+1$. 
Proof Recall that the Heegaard surface $\Sigma$ associated to the open book decomposition is gotten by doubling $P$ along its boundary, $\Sigma=P_{+1} \cup\left(-P_{-1}\right)$. We let $\alpha_{i}$ be the curve gotten by doubling $a_{i}, \alpha_{i}=a_{i} \cup \overline{a_{i}}$ and for the perturbed $\operatorname{arcs} b_{i}$ we get $\beta_{i}=b_{i} \cup \overline{\phi\left(b_{i}\right)}$.

Thus, the Heegaard diagram at $P_{+1} \subset \Sigma$ has a standard form (independent of the monodromy map). Our knot $L$ is adapted to the Heegaard diagram if it can be drawn on $P_{+1}$ so that it meets only $\alpha_{1}$ and $\beta_{1}$. The curve $L$ up to isotopy is in fact determined by any two points $w$ and $z$ in the two different components of $L-L \cap \alpha_{1}-L \cap \beta_{1}$. $L$ can be thought of now as a union of two arcs, $\xi$ crossing only $\alpha_{1}$ and $\eta$ crossing only $\beta_{1}$.

In the proof we will modify our Heegaard diagram in a way that it will accomodate the 0 -surgery along $L$, and hence the Seifert surface will be visible as a periodic domain. We stabilize $\Sigma$ once to obtain a new Heegaard diagram which corresponds to a Heegaard splitting with the property that $L$ is supported inside of the $\beta$-handlebody. Specifically, we let $\Sigma^{\prime}$ be the surface obtained by attaching a one-handle to $\Sigma$ with feet at $w$ and $z$. We introduce a new circle $\beta_{0}$ which is dual to the one-handle, and a circle $\alpha_{0}$ which meets $\beta_{0}$ at a single point, running through the one-handle, and completed by the arc $\eta$ outside the handle. The curve $\beta_{0}$ is a meridian for $L$. Let $\gamma_{0}$ be a circle which runs through the new one-handle so as not to meet $\alpha_{0}$, and runs along $\xi$ in the surface. See Figure 5 for an illustration.

The Heegaard diagram $\left(\Sigma^{\prime},\left\{\alpha_{0}, \ldots, \alpha_{g}\right\},\left\{\beta_{0}, \ldots, \beta_{g}\right\}\right)$ obtained by the above modification still represents $Y$. Moreover, $\left(\Sigma^{\prime},\left\{\alpha_{0}, \ldots, \alpha_{g}\right\},\left\{\gamma_{0}, \beta_{1}, \ldots, \beta_{g}\right\}\right)$ represents the three-manifold gotten by performing surgery on $L$ with its ThurstonBennequin framing. Other integral surgeries on $L$ are represented by replacing $\gamma_{0}$ by a suitable smoothing $\delta_{m}$ of $\gamma_{0}+m \beta_{0}$ with $m \in \mathbb{Z}$. The zero-surgery is characterized by the property that there is a periodic domain $\mathfrak{p}$, containing $\delta_{m}$ with multiplicity one along its boundary.

We claim that $\delta_{m}$ appears as a boundary component for a periodic domain in $\left(\Sigma^{\prime},\left\{\alpha_{0}, \ldots, \alpha_{g}\right\},\left\{\delta_{m}, \beta_{1}, \ldots, \beta_{g}\right\}\right)$ precisely when $m=n_{1}$ (in the notation of Lemma 4.5). We construct this periodic domain in three pieces, $A, B$, and $C$ which we define presently. Let $\bar{L}$ denote the copy of $L$ in $P_{-1} \subset \Sigma$. The two-chain $A$ is chosen so that

$$
\partial A-\alpha_{0}-\gamma_{0}+\bar{L} \in \operatorname{Span}\left(\left[\alpha_{i}\right]_{i=2}^{g}\right) ;
$$

the two-chain $B$ has the property that

$$
\partial B-\bar{L} \in \operatorname{Span}\left(\left[\beta_{0}\right],\left[\alpha_{i}-\beta_{i}\right]_{i=1}^{g}\right),
$$



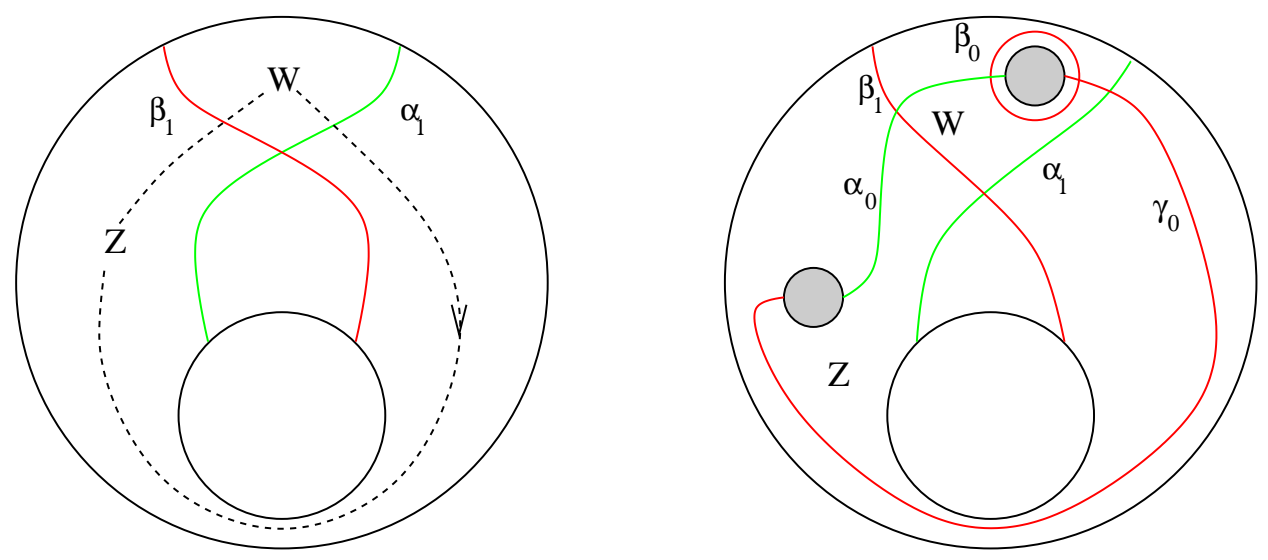

Figure 5: Stabilizing the Heegaard diagram for an open book. On the left, we have pictured $P_{+1}$, thought of as an annulus, with $L$ indicated by a dotted line (and the two basepoints $w$ and $z$ ). On the right, we have indicated a stabilization: the two grey circles represent the feet of a one-handle to be added. The curves $\alpha_{0}$ and $\gamma_{0}$ run through this one-handle.

hence gives a relation between $\bar{L}$, and a linear combination of $\beta_{0}$, and the $\left\{\alpha_{i}-\beta_{i}\right\}_{i=1}^{g}$. Finally, $C$ is a two-chain connecting $\beta_{0}, \gamma_{0}$, and $\delta_{m}$, i.e.

$$
\partial C=\delta_{m}-\gamma_{0}-m \beta_{0}
$$

see the picture in Figure 6 ,

The chain $A$ exists, as follows. Recall that $\Sigma^{\prime}$ is obtained by stabilizing $\Sigma=$ $P \cup(-P)$. We see that $\alpha_{0}+\gamma_{0}$ is homologous in $\Sigma^{\prime}$ to a copy of $L$, which we think of as supported in $\Sigma$ (i.e. it is supported away from the stabilization region in $\Sigma^{\prime}$ ). It suffices now to show that $L-\bar{L}$, thought of now as a curve in $\Sigma$, is homologous to a sum of the $\alpha_{i}$ (with $i>1$ ). We see this as follows. Cutting $P$ along $a_{i}$ with $i>2$, we end up with an annulus $X$ with some boundary arcs given by $a_{i}$, and containing $L$ as its core. Thus $L$ separates $P-a_{2}-\ldots-a_{g}$ into two components $C_{1}$ and $C_{2}$. Similarly, if we cut $\Sigma=P \cup(-P)$ along the $\alpha_{i}=a_{i} \cup \bar{a}_{i}(i>1)$, we see that the union of $L$ and $\bar{L}$ separates $\Sigma-\alpha_{2}-\ldots-\alpha_{g}$ into two components, $C_{1} \cup\left(-C_{1}\right)$, and $C_{2} \cup\left(-C_{2}\right)$. We let $A$ be the appropriate component (as determined by orientations).

The chain $B$ is constructed from the two-chain $\mathfrak{p}$ from Lemma 4.2, and by drilling out the disk with multiplicity $m$ in the region between $\alpha_{1}$ and $\beta_{1}$, i.e. $\partial B$ contains $\bar{L}$ with multiplicity one, $\alpha_{1}$ with multiplicity $-n_{1}$, and so contains $\beta_{0}$ with multiplicity $n_{1}$. The chain $C$ exists from the construction of $\delta_{m}$. 


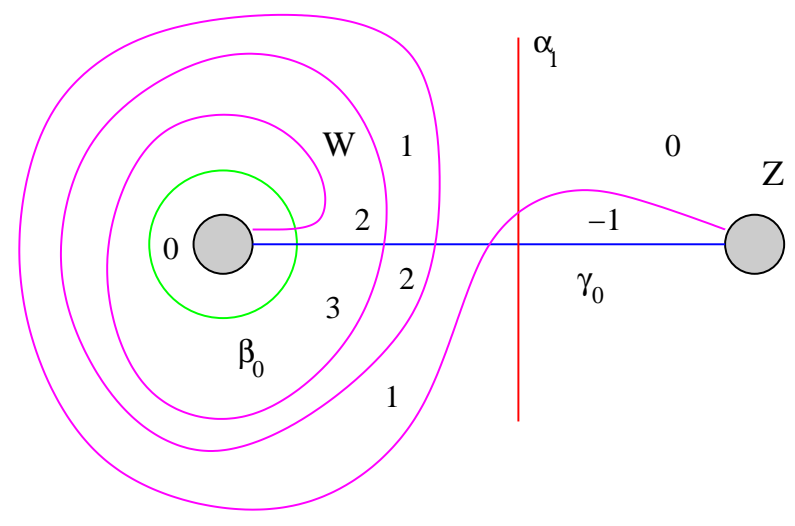

Figure 6: Two-chain of type $C$ when $m=3$. Its various local multiplicities are indicated.

The condition that $m=n_{1}$ ensures that, in the boundary of the sum $A+B+C$, the multiplicity of $\beta_{0}$ is zero. Since the multiplicity of $\gamma_{0}$ is also zero, we see that $A+B+C$ actually represents a periodic domain for the zero-surgery, as claimed.

Proof of Theorem 4.1 We think of

$$
\left(\Sigma,\left\{\delta_{m}, \beta_{1}^{\prime}, \ldots, \beta_{g}^{\prime}\right\},\left\{\beta_{0}, \ldots, \beta_{g}\right\}\left\{\alpha_{0}, \ldots, \alpha_{g}\right\}, z\right)
$$

(where $\beta_{i}^{\prime}$ are small isotopic translates of $\beta_{i}$ ) as a Heegaard triple representing the two-handle cobordism corresponding to the zero-surgery on $L \subset Y(\phi)$. Generators for $\mathbb{T}_{\beta} \cap \mathbb{T}_{\gamma}$ have "nearby" representatives in $\mathbb{T}_{\delta} \cap \mathbb{T}_{\alpha}$, as in Figure 7 . The Alexander grading of $\mathbf{x}$ can be calculated by the Alexander grading of the corresponding nearby point $\mathbf{x}^{\prime}$, since the two can be connected by a triangle $\psi \in \pi_{2}\left(\Theta, \mathbf{x}, \mathbf{x}^{\prime}\right)$ with $n_{w}(\psi)=n_{z}(\psi)=0$ (cf. [21, Section 2.3]). In turn, the Alexander grading of $\mathbf{x}^{\prime}$ is calculated with the help of the formula

$$
\left\langle c_{1}\left(\mathfrak{s}\left(\mathbf{x}^{\prime}\right)\right),[\widehat{F}]\right\rangle=2 p_{\mathbf{x}^{\prime}}(\widetilde{\mathfrak{p}})+e(\widetilde{\mathfrak{p}}),
$$

where here $\widetilde{\mathfrak{p}}$ is a periodic domain representing the homology class of $\widehat{F}$, and $p_{\mathbf{x}^{\prime}}(\widetilde{\mathfrak{p}})$ denotes the sum of the local multiplicities of $\widetilde{\mathfrak{p}}$ at the components of $\mathbf{x}^{\prime}$ cf. [23, Proposition 7.5]. As in the proof of Lemma 4.5, we construct a twochain representing a periodic domain in the zero-surgery, cut into three pieces $A, B$, and $C$ (in the notation from the previous proof). We claim that

$$
2 p_{\mathbf{x}^{\prime}}(A)+e(A)=0 .
$$




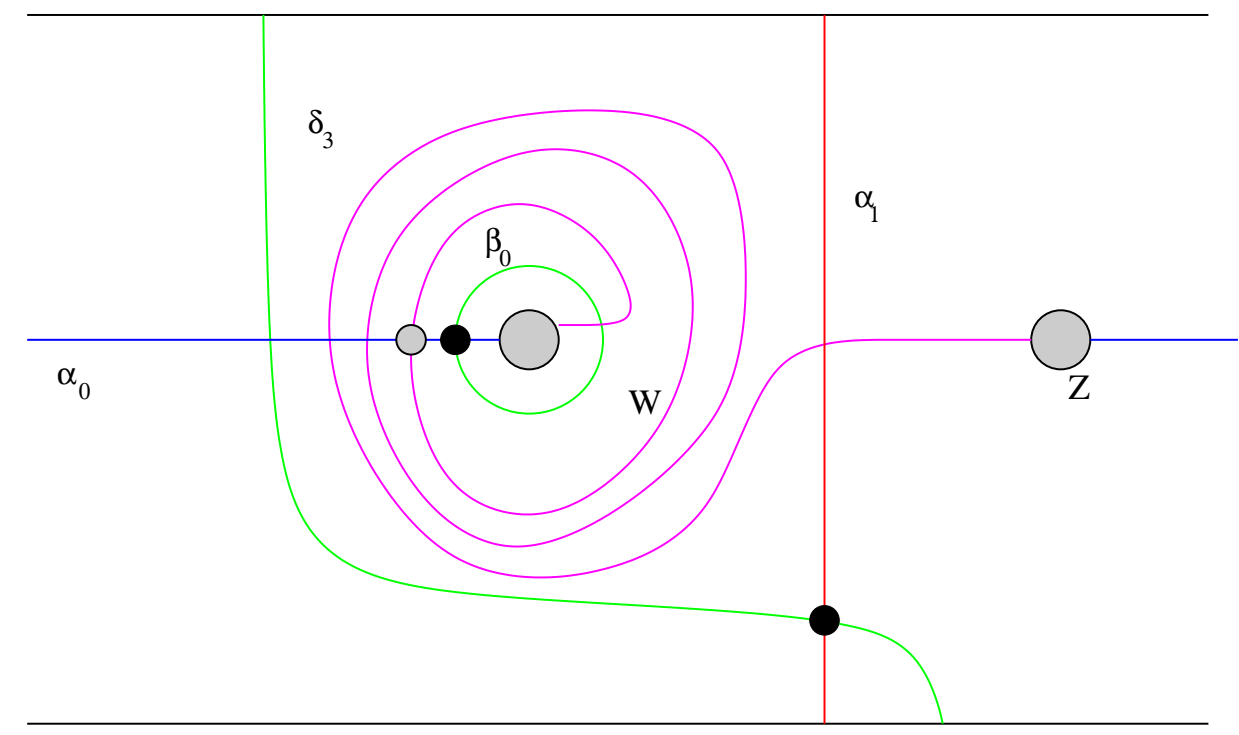

Figure 7: Nearby generator. The large grey circles represent the handle (compare Figure 5); the black circles represent the original generator (using $\left.\beta_{0}\right)$ while the grey one represents the surgery.

To see this, we argue as follows. Note that $p_{x_{0}}(A)=\frac{1}{2}$. There are three types of remaining component of $x_{i} \in \mathrm{x}^{\prime}$ with $i>0$ : those which are contained in the interior of $A$, those which are disjoint from the closure of $A$ (like $x_{1}$ ), and those which are contained in the boundary of $A$. At those which are not contained in the closure of $A, p_{x_{i}}(A)=0$. At those which are in the interior, $p_{x_{i}}(A)=1$. At those which are on the boundary, $p_{x_{i}}(A)=\frac{1}{2}$. But also it is easy to see that the Euler measure of $A$ is given by

$$
e(A)=-1-\#\left\{i \mid p_{x_{i}}(A)=\frac{1}{2}\right\}-2 \#\left\{i \mid p_{x_{i}}(A)=1\right\}
$$

indeed $A$ is a connected surface of genus $\#\left\{i \mid p_{x_{i}}(A)=1\right\}$, and whose number of boundary components is given by $3+\#\left\{i>0 \mid p_{x_{i}}(A)=\frac{1}{2}\right\}$. Equation (4.2) now follows.

We also claim that

$$
p_{\mathbf{x}^{\prime}}(B)=n_{1} .
$$

Indeed, $p_{x_{0}}(B)=n_{1}$ (since $x_{0}$ lies in the region between $\alpha_{1}$ and $\beta_{1}$ ), while for all $i>0, p_{x_{i}}(B)=0$ since at each $x_{i} \in \mathbf{x}^{\prime}$ which meets $B$, the four corners have local multiplicities $0, n_{i}, 0$, and $-n_{i}$. From the relationship between $B$ 
and $\mathfrak{p}$, it is clear that

$$
e(B)=-\left(e(\mathfrak{p})-n_{1}\right),
$$

since $B$ is obtained from $\mathfrak{p}$ by removing a disk with multiplicity $n_{1}$, and we evaluate on the $\left(-P_{-1}\right)$-side of the Heegaard surface (reversing the sign of the result).

Finally,

$$
e(C)=0 \quad \text { and } \quad p_{\mathbf{x}^{\prime}}(C)=-n_{1}+\frac{1}{2} .
$$

The first of these follows directly by inspecting the region $C$ (see Figure 6). For the second, observe that $p_{x_{i}^{\prime}}(C)=0$ except when $i=0$, in which case $p_{x_{0}^{\prime}}(C)=$ $-n_{1}+\frac{1}{2}$ (compare Figure 7, and the domain for $C$ pictured in Figure 6).

Combining Equations (4.2), (4.3), (4.4), and (4.5), we conclude that

$$
\left\langle c_{1}\left(\mathfrak{s}\left(\mathbf{x}^{\prime}\right)\right),[\widehat{F}]\right\rangle=e(\mathfrak{p})-n_{1}+1 .
$$

In view of Lemmas 4.4 and 4.5 , the theorem now follows.

Consider now the case of general ambient 3-manifold $Y$. In this case both the Alexander grading $A$ and the rotation number rot (as integer-valued invariants) require an additional choice: we need to fix a Seifert surface $F$ for $L$. Indeed, $A_{F}(\mathbf{x})$ is defined by the formula (4.1) with $\mathbf{t}=\mathbf{t}_{\mathbf{x}}$ being the relative $\operatorname{Spin}^{c}$ structure of $\mathbf{x}$, while $\operatorname{rot}_{F}(L)$ is the integral of the relative Euler class of the oriented 2-plane bundle $\xi$ on $F$, with the trivialization of $\xi_{\partial F}$ given by the oriented tangent vectors of $L$. With this choice, the quantities $A_{F}(\mathfrak{L}(L))$ and $\operatorname{rot}_{F}(L)$ are well-defined and we get

Theorem 4.6 For a fixed Seifert surface $F$ of the Legendrian knot $L \subset(Y, \xi)$ we have

$$
2 A_{F}(\mathfrak{L}(L))=\operatorname{tb}(L)-\operatorname{rot}_{F}(L)+1 .
$$

Proof In Lemma 4.2 we can choose the decomposition $[L]=\phi_{*}(Z)-Z$ in such a way that the Seifert surface resulting from $Z$ by Lemma 4.4 is homologous to $F$ in the relative homology group $H_{2}(Y, L)$. The rest of the proof is then applies verbatim. 


\subsection{Maslov gradings}

Equation (1.2) is much easier to establish. In fact, we establish the following more general version:

Theorem 4.7 Let $(Y, \xi)$ be a contact three-manifold with the property that $c_{1}(\xi)$ is a torsion homology class, so that $\widehat{\mathrm{CF}}(Y, \mathfrak{s}(\xi))$ has a rational Maslov grading, and also the two-plane field $\xi$ has a Hopf invariant $d_{3}(\xi) \in \mathbb{Q}$. Suppose moreover that $L \subset Y$ is a null-homologous Legendrian knot. Then, we have that

$$
2 A(\mathfrak{L}(L))-M(\mathfrak{L}(L))=d_{3}(\xi) .
$$

For more on the absolute (rational) Maslov grading on $\widehat{\mathrm{CF}}(Y, \mathbf{t})$ where $c_{1}(\mathbf{t})$ is a torsion class, see [20]. In defininig the Hopf invariant $d_{3}(\xi)$ of the $2-$ plane field underlying the contact structure $\xi$, we follow the convention used in 24]. Notice that when $c_{1}(\xi)$ is torsion, the Alexander grading $A(\mathfrak{L}(L))$ of the Legendrian invariant and the rotation number $\operatorname{rot}(L)$ of the Legendrian knot are independent of the chosen Seifert surface.

We can continue to think of $\mathrm{CFK}^{-}(Y, \mathbf{t})$ as a bigraded group, with a Maslov grading induced from $\mathrm{CF}^{-}(Y, \mathbf{t})$, and with an Alexander grading (which ordinarily we think of as given by relative $\operatorname{Spin}^{c}$ structures compatible with $\mathbf{t}$ ) defined as half the first Chern class of the relative $\operatorname{Spin}^{c}$ structure evaluated on a Seifert surface for $K$. This latter quantity will be denoted by $s$.

Consider the map

$$
F: \mathrm{CFK}^{-}(-Y, L, \mathbf{t}, s) \longrightarrow \widehat{\mathrm{CF}}(-Y, \mathbf{t})
$$

gotten by setting $U=1$, and then viewing $z$ as the basepoint for $\widehat{\mathrm{CF}}(-Y, \mathbf{t})$.

Proposition 4.8 The map $F$ sends $\mathrm{CFK}_{d}^{-}(-Y, L, \mathbf{t}, s) \longrightarrow \widehat{\mathrm{CF}}_{d-2 s}(-Y, \mathbf{t})$.

Proof For each given $s$, the map $F$ clearly preserves the relative Maslov grading, as that is given by $\mu(\phi)$ where $\phi \in \pi_{2}(\mathbf{x}, \mathbf{y})$ satisfies $n_{w}(\phi)=n_{z}(\phi)=$ 0 . Moreover, if $\phi \in \pi_{2}(\mathbf{x}, \mathbf{y})$ satisfies $n_{w}(\phi)=0$ and $n_{z}(\phi)=k$, so that $A(\mathbf{x})-A(\mathbf{y})=k$, then under the specialization, we see that $\phi$ drops Maslov grading by $2 k$. It follows at once that there is a constant $c$ with the property that $F$ sends $\mathrm{CFK}_{d}^{-}(-Y, L, \mathbf{t}, s)$ to $\widehat{\mathrm{CF}}_{d-2 s-c}(-Y, \mathbf{t})$. The fact $c=0$ follows from conjugation symmetry of Floer homology. 
Proof of Theorem 4.7. The construction of the Legendrian invariant $\mathfrak{L}(L)$ implies that $F(\mathfrak{L}(L))=c(Y, \xi)$, where here $c(Y, \xi) \in \widehat{\mathrm{CF}}(-Y)$ is the contact invariant from [24]. By 24] the Maslov grading of the contact invariant is $M(c(\xi))=-d_{3}(\xi)$, hence Proposition 4.8 above implies that $M(\mathfrak{L}(L))$ $2 A(\mathfrak{L}(L))=-d_{3}(\xi)$, concluding the proof.

Proof of Theorem 1.6 Theorem 1.6 is now a combination of Theorems 4.1 and 4.7.

\section{$5 \quad$ Transverse simplicity of knots in $\left(S^{3}, \xi_{s t}\right)$}

\subsection{The Eliashberg-Chekanov knots}

We will demonstrate the power of the transformation rule proved in Section 3 by computing the invariants of the Eliashberg-Chekanov Legendrian knots and verify Theorem 1.3. To this end, consider the Legendrian knot $E(k, l)$ given by Figure 8 .

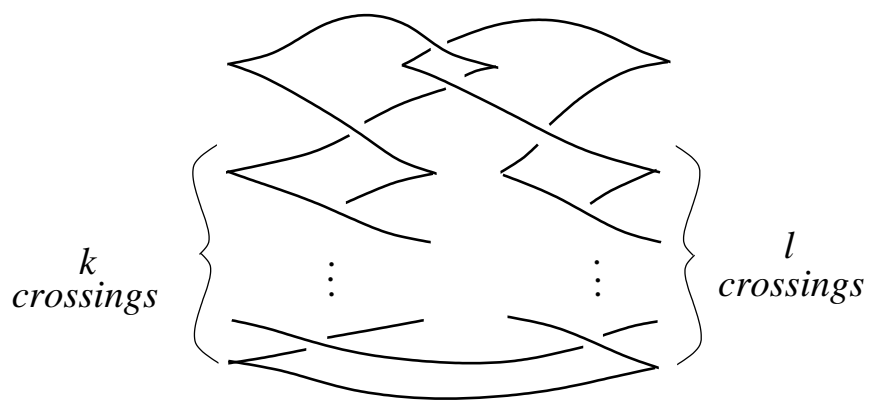

Figure 8: The Legendrian knots $E(k, l)$. These knots are smoothly isotopic to $E_{n}$, with $k+l-1=n$.

Proposition 5.1 The knot $E(k, l)$ is a Legendrian knot in the standard contact 3-sphere smoothly isotopic to the Eliashberg-Cheknov knot $E_{n}$ with $k+l-1=n, c f$. [5]. The Thurston-Bennequin and rotation numbers of $E(k, l)$ are given as $\operatorname{tb}(E(k, l))=1$ and $\operatorname{rot}(E(k, l))=0$.

Corollary 5.2 The Legendrian invariant $\mathfrak{L}(E(k, l))$ is a nonzero element of the knot Floer homology group $\operatorname{HFK}_{2}^{-}\left(-S^{3}, E_{n}, 1\right)$. 
Proof According to the nonvanishing of the invariant in a Stein fillable contact 3-manifold, we get $\mathfrak{L}(E(k, l)) \neq 0$. The Alexander and Maslov gradings of $\mathfrak{L}(E(k, l))$ can be computed from the rotation and Thurston-Bennequin numbers (given in Proposition 5.1) through the formulae of Theorem 1.6.

Let $k=\frac{n+1}{2}$. The knot Floer homology group $\operatorname{HFK}^{-}\left(-S^{3}, E_{n}\right)=\operatorname{HFK}^{-}\left(S^{3}, m\left(E_{n}\right)\right)=$ $\operatorname{HFK}^{-}\left(m\left(E_{n}\right)\right)$ for $n \geq 1$ and odd is given as

$$
\operatorname{HFK}_{M}^{-}\left(m\left(E_{n}\right), A\right)= \begin{cases}\mathbb{F}^{k} & (A=1, M=2) \\ \mathbb{F}^{k} & (A=0, M=1) \\ \mathbb{F} & (A=-i \leq 0, M=-2 i) .\end{cases}
$$

Also, $\widehat{\operatorname{HFK}}\left(m\left(E_{n}\right)\right)$ (which can be read off directly from the Alexander polynomial and the signature of the knot since $E_{n}$ is an alternating knot, see [18, 26]) is given as

$$
\widehat{\operatorname{HFK}}_{M}\left(m\left(E_{n}\right), A\right)= \begin{cases}\mathbb{F}^{k} & (A=1, M=2) \\ \mathbb{F}^{n} & (A=0, M=1) \\ \mathbb{F}^{k} & (A=-1, M=0) .\end{cases}
$$

Corollary 5.3 The Legendrian invariant $\widehat{\mathfrak{L}}(E(k, l))$ is a nonzero element of the knot Floer group $\widehat{\operatorname{HFK}}\left(m\left(E_{n}\right)\right)$.

Proof The explicit description of the specialization map $\operatorname{HFK}^{-}\left(m\left(E_{n}\right)\right) \rightarrow$ $\widehat{\operatorname{HFK}}\left(m\left(E_{n}\right)\right.$ ) (when setting $U=0$ ) and the fact that the invariant lives in bidegree $(A=1, M=2)$ readily implies the corollary.

We would like to verify that the Legendrian $\operatorname{knots} E(k, l)$ with $k+l-1=n$, $k, l$ odd and $k \leq l$ have different Legendrian invariants. As usual, we use $\widehat{\mathrm{HFK}}$, implying the similar distinction result for the invariants in the $\mathrm{HFK}^{-}$-groups.

Theorem 5.4 Let us fix a knot $E_{n} \subset S^{3}$. There are identifications of $\widehat{\operatorname{HFK}}\left(S^{3}, m(E(k, l))\right)$ with $\widehat{\operatorname{HFK}}\left(S^{3}, m\left(E_{n}\right)\right)$, for $k+l-1=n, k, l$ odd such that the images of the the Legendrian invariants $\widehat{\mathfrak{L}}(E(k, l))$ and $\widehat{\mathfrak{L}}\left(E\left(k^{\prime}, l^{\prime}\right)\right)$ are equal in $\widehat{\operatorname{HFK}}\left(S^{3}, m\left(E_{n}\right)\right)$ if and only if $k=k^{\prime}$ and $l=l^{\prime}$.

The action of the mapping class group taken into account, this statement gives

Proof of Theorem 1.3 Since $E_{n}$ is a two-bridge knot, which is not a torus knot, and by [8] all non-torus 2-bridge knots are hyperbolic, [28, Theorem 2.7] implies that the mapping class group $\operatorname{MCG}\left(S^{3}, E_{n}\right)$ is isomorphic to $\mathbb{Z} / 2 \mathbb{Z}$. The 
Legendrian knots $E(k, l)$ and $E(l, k)$ are Legendrian isotopic [5, Theorem 4.1], hence under the action of the mapping class group the element $\widehat{\mathfrak{L}}(E(k, l))$ is mapped to $\widehat{\mathfrak{L}}(E(l, k))$. This identifies the $\mathbb{Z} / 2 \mathbb{Z}$-action of $\operatorname{MCG}\left(S^{3}, E_{n}\right)$ on the knot Floer homology group $\widehat{\operatorname{HFK}}_{2}\left(m\left(E_{n}\right), 1\right)$, and shows that $\widehat{\mathfrak{L}}(E(k, l))$ and $\widehat{\mathfrak{L}}\left(E\left(k^{\prime}, l^{\prime}\right)\right)$ are in different $\operatorname{MCG}(Y, L)$-orbits, provided $k, l, k^{\prime}, l^{\prime}$ are odd, $k+l-1=k^{\prime}+l^{\prime}-1=n, k \leq l, k^{\prime} \leq l^{\prime}$ and $k \neq k^{\prime}$. This shows that the corresponding Legendrian knots and their negative stabilizations are not isotopic, concluding the proof of the theorem.

Remark 5.5 Notice that the above result, in conjunction with [5, Theorems 2.2 and 4.2] shows that among the knots given by positive transverse push-offs of the diagram of Figure 8 there are exactly $\left\lceil\frac{n}{4}\right\rceil$ transversely nonisotopic. It is still an open question whether there are further transverse representatives of $E_{n}$ with self-linking number 1 not transverse isotopic to any of the transverse push-offs of the Legendrian knots considered above.

For the proof of Theorem 5.4, consider the 2-component Legendrian link of Figure 9. Notice that the linking number of the two knots is zero. The smooth
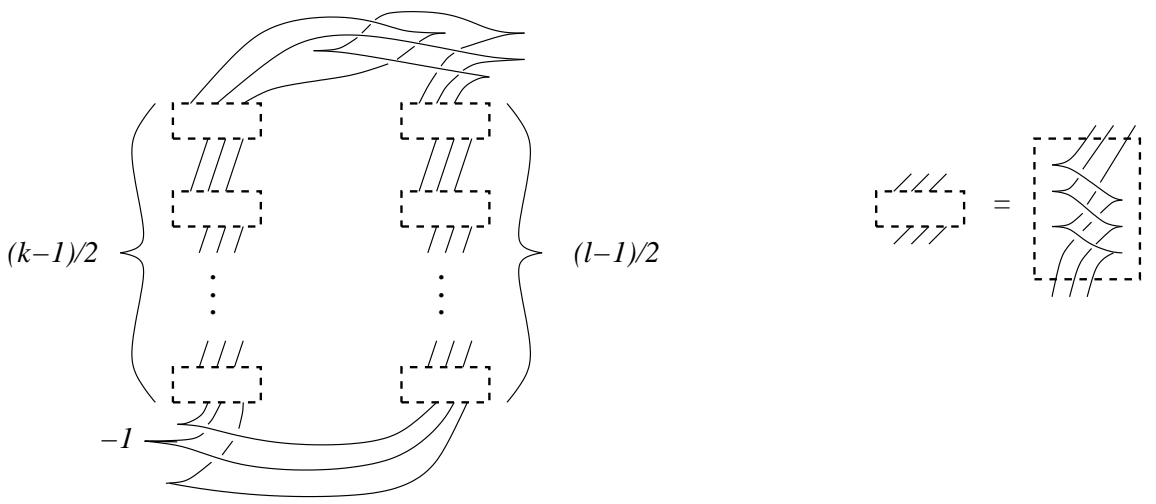

Figure 9: The Legendrian knot $E(k, l)$ together with an unknot.

diagram underlying Figure 9 is given by Figure 10. Applying contact $(-1)-$ surgery on the unknot component of Figure 9, we get a Legendrian knot $E^{\prime}(k, l)$ in the lens space $L(m+1,1)$ with contact structure $\xi_{k, l}$. Let $S$ denote the Legendrian push-off of the unknot in Figure 9. It is a standard fact in contact topology that contact $(+1)$-surgery along $S$ cancels the first contact $(-1)$ surgery, and hence provides the standard contact 3-sphere with the Legendrian knot $E(k, l)$ in it. The surgery along $S$ induces the map

$$
\widehat{F}_{S}: \widehat{\operatorname{HFK}}\left(-L(m+1,1), E^{\prime}(k, l)\right) \rightarrow \widehat{\operatorname{HFK}}\left(-S^{3}, E(k, l)\right) .
$$




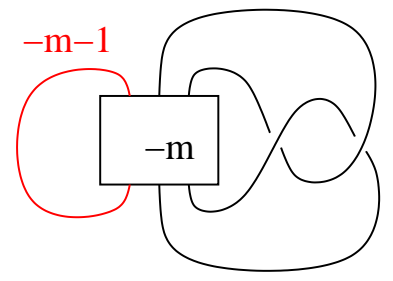

Figure 10: The smooth knots of Figure 9. We let $m=\frac{n+1}{2}$.

(Notice the orientation reversal on the 3 -manifolds.)

Proposition 5.6 The Legendrian invariants $\widehat{\mathfrak{L}}\left(E^{\prime}(k, l)\right)$ are all distinct, and the map $\widehat{F}_{S}$ is injective on the subgroup of the knot Floer homology with Alexander grading 1.

Proof The surgery along $S$, with orientation reversed, gives rise to a distinguished triangle of knots, depicted in Figure 11, For simplicity, here we slid the surgery curve $S$ over the unknot to which it is a Legendrian push-off, resulting in a meridional unknot with framing 0. As it was explained in Subsection 2.3, such a distinguished triangle of knots induces an exact triangle on the knot Floer homology groups. In addition, since the surgery curve and the knot under inspection gives zero linking, we can consider groups only with a fixed Alexander grading, since all maps do respect that Alexander gradings. Since $\widehat{\mathfrak{L}}(L)$ is a nonzero element with Alexander grading 1 , we examine the $A=1$ groups only.

The third term of the triangle is an unknot in the lens space $-L(m, 1)$, therefore at Alexander grading 1 the corresponding knot Floer group vanishes, implying that the map $\widehat{F}_{S}$ is an isomorphism on that particular Alexander grading.

The element $\mathfrak{L}\left(E^{\prime}(k, l)\right)$ specializes to $c\left(L(m+1,1), \xi_{k, l}\right)$ under the specialization $U=1$. Since $\xi_{k, l}$ and $\xi_{k^{\prime}, l^{\prime}}$ induce the same $\operatorname{Spin}^{c}$ structure only if $k=k^{\prime}$ and $l=l^{\prime}$, we conclude that the invariants $\mathfrak{L}\left(E^{\prime}(k, l)\right)$ are different for different $k$ 's.

Proof of Theorem 5 The injectivity of $\widehat{F}_{S}$ and the fact that all $\widehat{\mathfrak{L}}\left(E^{\prime}(k, l)\right)$ are different, together with the naturality formula

$$
\widehat{F}_{S}\left(\widehat{\mathfrak{L}}\left(E^{\prime}(k, l)\right)=\widehat{\mathfrak{L}}(E(k, l))\right.
$$

concludes the proof. 


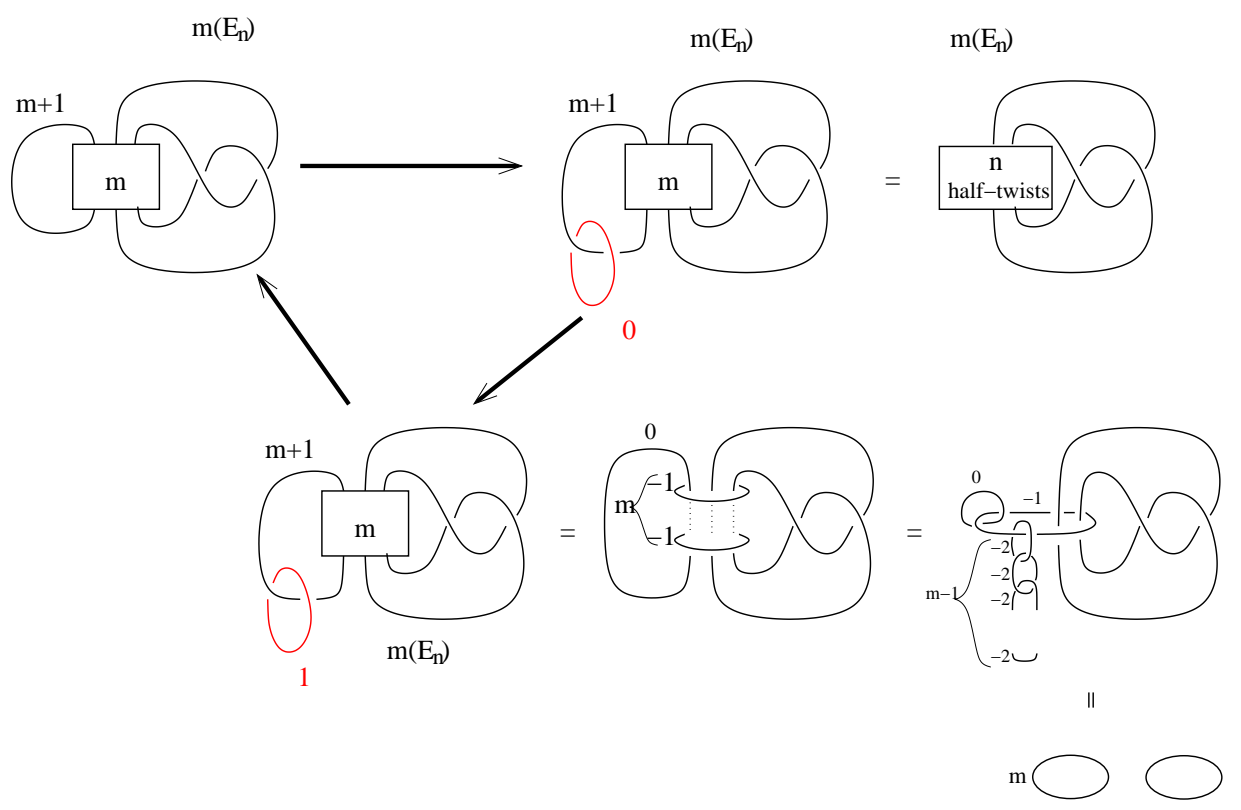

Figure 11: The distinguished triangle of knots induced by the surgery along $S$ (after the reversal of orientation). Recall that $m=\frac{n+1}{2}$.

\subsection{Two-bridge knots}

The method of the above argument can be generalized to find further examples of knot types containing distinct transverse knots with identical self-linking numbers. Here we formulate a result exploiting the same ideas used above, and then provide some further families where this principle can be used. The candidates will be constructed through the Legendrian satellite construction described in [16] - in fact, the Eliashberg-Chekanov knots considered above are special cases of this construction.

Let us start by recalling the Legendrian satellite construction. To this end, let $\tilde{L}$ denote a Legendrian link in $S^{1} \times \mathbb{R}^{2}$, which can be conveniently depicted by cutting $S^{1}$ open at a point, hence $\tilde{L}$ can be drawn in a box, cf. [16, Figure 22]. Now for a Legendrian knot $L$ consider its standard neighbourhood. By an appropriate contactomorphism between this solid torus and the one containing $\tilde{L}$ we can embed $\tilde{L}$ into the neighbourhood of $L$. We define $S(L, \tilde{L})$ as this new Legendrian knot. If $w(\tilde{L})$ denotes the winding number of $\tilde{L}$ in $S^{1} \times \mathbb{R}^{2}$, then we have

$$
\operatorname{tb}(S(L, \tilde{L}))=(w(\tilde{L}))^{2} \operatorname{tb}(L)+\operatorname{tb}(\tilde{L})
$$


and

$$
\operatorname{rot}(S(L, \tilde{L}))=(w(\tilde{L}))^{2} \operatorname{rot}(L)+\operatorname{rot}(\tilde{L}) .
$$

Consequently, in case $w(\tilde{L})=0$, the Thurston-Bennequin and the rotation numbers of $S(L, \tilde{L})$ are independent of $L$.

Suppose that $\tilde{L}$ is a Legendrian solid-torus knot with $w(\tilde{L})=0$, and $U(a, b)$ is a Legendrian realization of the unknot, with $\operatorname{tb}(U(k, l))=-1-(a+b)$ and $\operatorname{rot}(U(a, b))=a-b$. Let $L_{k, l}=S(U(a, b), \tilde{L})$ denote the Legendrian satellite of $U(a, b)$ with $\tilde{L}$, where here $k=2 a+1$ and $l=2 b+1$. Let $K_{0}$ denote the knot (in some lens space) given by the sugery description of Figure 12, Let

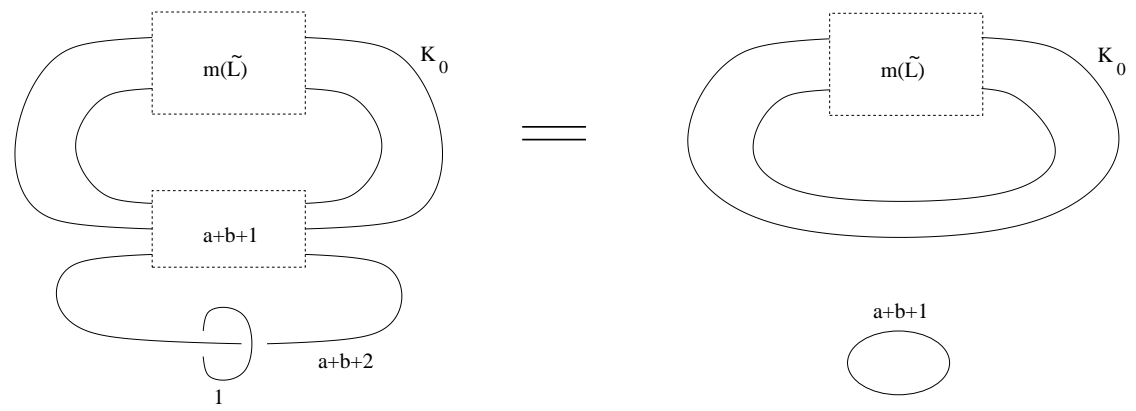

Figure 12: The third knot $K_{0}$ in the distinguished triangle of knots.

$g\left(K_{0}\right)$ denote genus of the knot $K_{0}$. (By [17], this quantity bounds the largest Alexander grading with nontrivial knot Floer homology in $\operatorname{HFK}^{-}\left(Y, K_{0}\right)$.)

Theorem 5.7 Let $k=2 a+1$ and $l=2 b+1$. If $\operatorname{tb}(\tilde{L})+\operatorname{rot}(\tilde{L})>2 g\left(K_{0}\right)-1$ and the symmetry group of the smooth knot underlying $L_{k, l} \subset S^{3}$ is of order $t$ then the knot type of $L_{k, l}$ admits at least $\left\lceil\frac{k+l-1}{2 t}\right\rceil$ transversely non-isotopic transverse representatives.

Proof The same set-up as for the Eliashberg-Chekanov knots provides the Legendrian knots $L_{k, l}^{\prime}$ in the lens space $L(a+b+2,1)$, and the map induced by the $(+1)$-surgery is an isomorphism again, since in the surgery triangle the third term vanishes. This vanishing is because in the Alexander grading $A=$ $\frac{1}{2}\left(\operatorname{tb}\left(L_{k, l}\right)+\operatorname{rot}\left(L_{k, l}\right)+1\right)=\frac{1}{2}(\operatorname{tb}(\tilde{L})+\operatorname{rot}(\tilde{L})+1)$ the knot Floer homology group of $K_{0}$ vanishes by the assumption. Now the analogue of Proposition 5.6 provides different invariants before the action of the mapping class group $\operatorname{MCG}\left(S^{3}, L_{k, l}\right)$ is taken into account. Since in $\widehat{\operatorname{HFK}}\left(S^{3}, m\left(L_{k, l}\right)\right) / \operatorname{MCG}\left(S^{3}, L_{k, l}\right)$ we will still have at least $\left\lceil\frac{k+l-1}{2 t}\right\rceil$ different invariants, the proof follows. 
A simple example of $\tilde{L}$ with $w(\tilde{L})=0$, tb $=1$ and $K_{0}=$ Unknot is given by Figure 13. Notice that the orientation depicted in the figure implies that there are an even number of crossings in the projection. Since the knots $S(U(a, b), \tilde{L})$

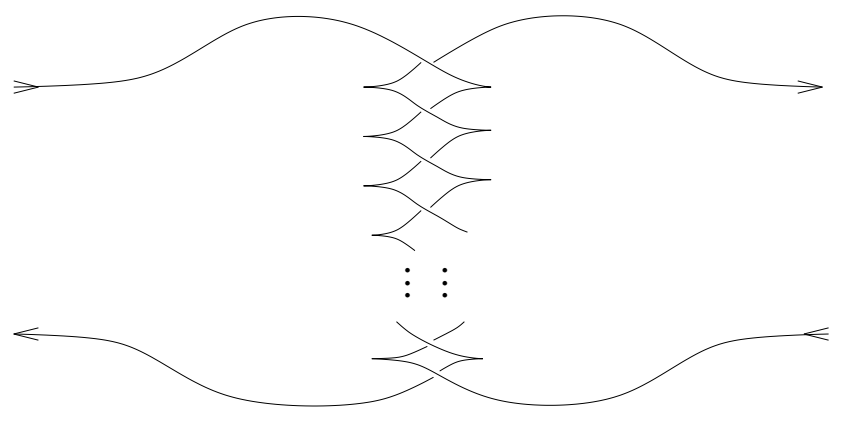

Figure 13: Further examples.

are all 2-bridge knots, and those knots have small symmetry groups provided they are not torus knots, we find further examples of transversely non-simple families of knot types.

There are various ways to further generalize this construction. For example, the top crossing of Figure 13 can be replaced with a sequence of crossings, as it is shown in Figure 14, In order to get a knot, rather than a link, we require

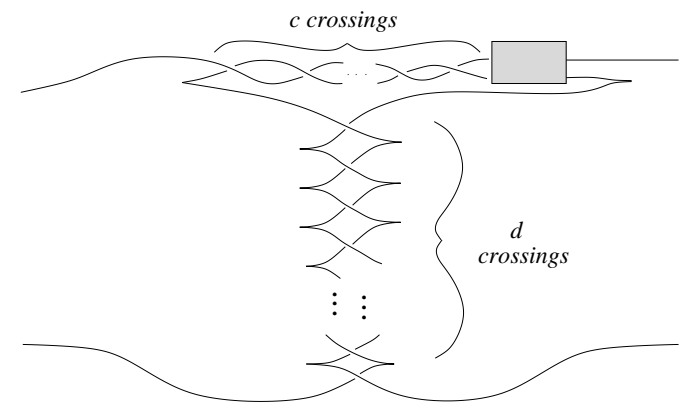

Figure 14: Iterating the construction

the parity of the number $c$ of these new crossings to be odd. The ThurstonBennequin number of the knot $S(U(a, b), \tilde{L})$ with $\tilde{L}$ given by Figure 14 (with strands simply passing through the gray box) can be easily computed to be equal to $c$, while the Euler characteristic of a Seifert surface of $K_{0}$ appearing in Theorem 5.7 is $1-c$. (Notice that $K_{0}$ is, in fact, isotopic to the $(2, c)$ torus knot.) Since $L_{k, l}=S(U(a, b), \tilde{L})$ with $k=2 a+1$ and $l=2 b+1$ for $k+l>2$ is 
a 2-bridge knot which is not a torus knot, its mapping class group is known to be isomorphic to $\mathbb{Z} / 2 \mathbb{Z}$, hence Theorem 5.7 shows that the knot types appearing in this construction (with $k+l>2$, both odd) are not transversely simple. The above construction admits a further generalization as follows:

Theorem 5.8 Suppose that $\frac{p}{q} \in \mathbb{Q}$ has the continued fractions expansion

$$
\frac{p}{q}=\left[a_{1}, \ldots, a_{2 m+1}\right]=a_{1}+\frac{1}{a_{2}+\frac{1}{\ddots \cdot \frac{1}{a_{2 m+1}}}},
$$

and suppose that

- $a_{2}$ and $a_{3}$ are odd,

- all $a_{i}$ for $i \neq 2,3$ are even and

- $m \geq 1$.

Let $K=K(p, q)$ be the corresponding two-bridge knot. Then, $K$ admits at least $\left\lceil\frac{a_{1}}{4}\right\rceil$ distinct transverse realizations with self-linking number sl equal to

$$
\sum_{i=1}^{m} a_{2 i+1}
$$

Proof Consider the sequence $\widetilde{L}_{i}(i=1, \ldots, m)$ of solid torus knots given by Figure 14, where the parameters $c_{i}, d_{i}$ are chosen so that $c_{1}, d_{1}$ are both odd, while $c_{i}, d_{i}$ are even for $i>1$. Starting with $i=m$, copy $\widetilde{L}_{i}$ into the gray box of $\widetilde{L}_{i-1}$. The parity assumptions on $c_{i}, d_{i}$ ensure that $\widetilde{L}_{i}$ can be drawn in the indicated box of $\widetilde{L}_{i-1}$ with consistent orientations. At the end of this process we get a solid torus link $\widetilde{L}$, with $w(\widetilde{L})=0$. We use it to define the set of Legendrian knots $L_{k, l}=S(U(a, b), \widetilde{L})$ with $k=2 a+1, l=2 b+1$ as before.

To calculate the Thurston-Bennequin number, we argue as follows. According to Equation (5.1) $\operatorname{tb}(S(L, \widetilde{L}))=\operatorname{tb}(\widetilde{L})$. To calculate this, observe first that all crossings in the diagram are positive; hence the writhe is the total number of crossings. Moreover, in the $\widetilde{L}_{i}$ template, the $d_{i}$ crossings cancel with the left cusps, leaving only the $c_{i}$ to contribute to the Thurston-Bennequin invariant. Consequently,

$$
\operatorname{tb}\left(L_{k, l}\right)=\sum_{i=1}^{m} c_{i}
$$

Applying Seifert's algorithm, we see that the negative of the Euler characteristics of a Seifert surface for the knot $K_{0}$ appearing in Theorem 5.7 is equal to 
$\left(\sum_{i=1}^{m} c_{i}\right)-1$. The rotation number $\operatorname{rot}(\widetilde{L})$ is zero, therefore by Equation (5.2), we see that $\operatorname{rot}\left(L_{k, l}\right)=0$ as well. This implies that the self-linking number $\operatorname{sl}\left(T_{k, l}\right)$ of the transverse push-off of $L_{k, l}$ is equal to $\sum_{i=1}^{m} c_{i}$.

In fact, it is easy to see that $L_{k, l}$ is the two-bridge knot $K(p, q)$ with $\frac{p}{q}=$ $\left[a_{1}, \ldots, a_{2 m+1}\right]$, where $a_{1}=k+l, a_{2 i}=d_{i}, a_{2 i+1}=c_{i}$. Since $m \geq 1$, this is not a torus knot. Thus, applying [8] and [28] as before, we conclude that the mapping class group has order two. Hence, Theorem 5.7 provides the stated result.

\section{Mapping class group actions}

We construct here the mapping class group action on knot Floer homology. Our discussion here is built on the constructions from [25] (which dealt, however, with Heegaard Floer homology for closed three-manifolds), combined with [21].

A marked Heegaard diagram for a pointed knot $(Y, K, p)$ is a Heegaard diagram

$$
\left(\Sigma,\left\{\alpha_{1}, \ldots, \alpha_{g}\right\},\left\{\beta_{1}, \ldots, \beta_{g}\right\}, w, z\right)
$$

for $Y$ so that $w$ and $z$ determine $K$, and $w$ corresponds to the marked point $p \in K$. We can associate a Heegaard Floer complex $\mathrm{CFK}^{-}(\Sigma, \alpha, \beta, w, z)$ to this doubly-pointed Heegaard diagram, provided that it satisfies a suitable weak admissibility condition, see [23].

Proposition 6.1 Suppose that $\left(\Sigma^{1}, \alpha^{1}, \beta^{1}, w^{1}, z^{1}\right)$ and $\left(\Sigma^{2}, \alpha^{2}, \beta^{2}, w^{2}, z^{2}\right)$ are two marked Heegaard diagrams for $(Y, K)$. Then, these two diagrams can be connected by Heegaard moves in the sense that there are the following data:

- marked diagrams $\left(\Sigma, \alpha^{3}, \beta^{3}, w, z\right)$ and $\left(\Sigma, \alpha^{4}, \beta^{4}, w, z\right)$ obtained by stabilizing $\left(\Sigma^{1}, \alpha^{1}, \beta^{1}, w^{1}, z^{1}\right)$ and $\left(\Sigma^{2}, \alpha^{2}, \beta^{2}, w^{2}, z^{2}\right)$ respectively;

- a sequence of handleslides and isotopies from $\left(\Sigma, \alpha^{3}, \beta^{3}, w, z\right)$ to $\left(\Sigma, \alpha^{4}, \beta^{4}, w, z\right)$ respectively, so that none of the $\alpha$-curves crosses either $w$ or $z$ during the handleslides and isotopies.

Proof This is a modification of the usual Reidemeister-Singer theorem, stating that two Heegaard diagrams for $Y$ can be connected by stabilizations, destabilizations, handleslides, and isotopies. We fix a Morse function $f_{0}$ near $K$, with one index 3 and one index 0 critical point on $K$, and consider extensions of this fixed Morse function to $Y$. 
Consider the following definition (the map defined by a strong equivalence in the sense of [23, Lemma 2.13]).

Definition 6.2 Suppose that $\left(\Sigma, \alpha^{3}, \beta^{3}, w, z\right)$ and $\left(\Sigma, \alpha^{4}, \beta^{4}, w, z\right)$ are admissible Heegaard diagrams for $Y$ which differ only by handleslides and isotopies. Let $\alpha^{5}, \beta^{5}$ be isotopic translates of the $\alpha^{3}, \beta^{3}$, so that $\left(\Sigma, \alpha^{5}, \beta^{5}, w, z\right)$ is also an admissible diagram for $Y$, and $\left(\Sigma, \alpha^{4}, \alpha^{5}, w, z\right)$ and $\left(\Sigma, \beta^{5}, \beta^{4}, w, z\right)$ are both weakly admissible Heegaard diagrams for $\#^{g}\left(S^{2} \times S^{1}\right)$. We define a map, up to overall multiplication by \pm 1 ,

$$
\Phi_{3,4}: \mathrm{CFK}^{-}\left(\Sigma, \alpha^{3}, \beta^{3}, w, z\right) \longrightarrow \mathrm{CFK}^{-}\left(\Sigma, \alpha^{4}, \beta^{4}, w, z\right)
$$

as a composite

$\mathrm{CFK}^{-}\left(\Sigma, \alpha^{3}, \beta^{3}, w, z\right) \stackrel{\Gamma}{\longrightarrow} \mathrm{CFK}^{-}\left(\Sigma, \alpha^{5}, \beta^{5}, w, z\right) \stackrel{\Theta_{\alpha^{4} \alpha^{5} \otimes \cdot \otimes \Theta_{\beta^{5} \beta^{4}}}^{\longrightarrow}}{\longrightarrow} \mathrm{CFK}^{-}\left(\Sigma, \alpha^{4}, \beta^{4}, w, z\right)$,

where here the first map is induced by isotopies, and defined using continuation maps (i.e. counting holomorphic disks with time-dependent boundary conditions), while the second is defined by counting pseudo-holomorphic trianlges, cf. [25, Section 2.3].

Given any two marked Heegaard diagrams $\left(\Sigma^{1}, \alpha^{1}, \beta^{1}, w^{1}, z^{1}\right)$ and $\left(\Sigma^{2}, \alpha^{2}, \beta^{2}, w^{2}, z^{2}\right)$, for suitable choices of almost-complex structure, the stabilization/destabilization maps give identifications

$$
\begin{aligned}
& f_{1,3}: \operatorname{CFK}^{-}\left(\Sigma^{1}, \alpha^{1}, \beta^{1}, w^{1}, z^{1}\right) \longrightarrow \operatorname{CFK}^{-}\left(\Sigma, \alpha^{3}, \beta^{3}, w, z\right) \\
& f_{4,2}: \operatorname{CFK}^{-}\left(\Sigma, \alpha^{4}, \beta^{4}, w, z\right) \longrightarrow \operatorname{CFK}^{-}\left(\Sigma^{2}, \alpha^{2}, \beta^{2}, w^{2}, z^{2}\right) .
\end{aligned}
$$

Define the map $\Psi_{1,2}$ by $f_{4,2} \circ \Phi_{3,4} \circ f_{1,3}$. According to the following variant of [23, Theorem 2.1], the induced map

$$
\left(\Psi_{1,2}\right)_{*}: \operatorname{HFK}^{-}\left(\Sigma^{1}, \alpha^{1}, \beta^{1}, w^{1}, z^{1}\right) \longrightarrow \operatorname{HFK}^{-}\left(\Sigma^{2}, \alpha^{2}, \beta^{2}, w^{2}, z^{2}\right)
$$

is independent of all the choices made; that is

Theorem 6.3 ([23, Theorem 2.1]) If $\left(\Sigma^{1}, \alpha^{1}, \beta^{1}, w^{1}, z^{1}\right)$ and $\left(\Sigma^{2}, \alpha^{2}, \beta^{2}, w^{2}, z^{2}\right)$ are two marked Heegaard diagrams for $(Y, K)$, then the connecting Heegaard moves from Proposition 6.1 induce a chain map

$$
\Psi_{1,2}: \mathrm{CFK}^{-}\left(\Sigma^{1}, \alpha^{1}, \beta^{1}, w^{1}, z^{1}\right) \longrightarrow \mathrm{CFK}^{-}\left(\Sigma^{2}, \alpha^{2}, \beta^{2}, w^{2}, z^{2}\right)
$$

whose chain homotopy class, up to multiplication by \pm 1 , is independent of the intermediate stages. 
Definition 6.4 A homeomorphism of Heegaard diagrams is a map

$$
f:\left(\Sigma^{1}, \alpha^{1}, \beta^{1}, w^{1}, z^{1}\right) \longrightarrow\left(\Sigma^{2}, \alpha^{2}, \beta^{2}, w^{2}, z^{2}\right)
$$

which is a homeomorphism from $\Sigma^{1}$ to $\Sigma^{2}$, carrying the set $\alpha^{1}=\left\{\alpha_{j}^{1}\right\}_{j=1}^{g}$ to the set $\alpha^{2}=\left\{\alpha_{j}^{2}\right\}_{j=1}^{g}, \beta^{1}$ to $\beta^{2}, w^{1}$ to $w^{2}$, and $z^{1}$ to $z^{2}$.

A homeomorphism

$$
f:\left(\Sigma^{1}, \alpha^{1}, \beta^{1}, w^{1}, z^{1}\right) \longrightarrow\left(\Sigma^{2}, \alpha^{2}, \beta^{2}, w^{2}, z^{2}\right)
$$

of Heegaard diagrams induces a continuous map from $\operatorname{Sym}^{g}\left(\Sigma^{1}\right)$ to $\operatorname{Sym}^{g}\left(\Sigma^{2}\right)$ carrying $\mathbb{T}_{\alpha^{1}}$ and $\mathbb{T}_{\beta^{1}}$ to $\mathbb{T}_{\alpha^{2}}$ and $\mathbb{T}_{\beta^{2}}$ respectively. This induces a map of chain complexes

$$
\Xi_{1,2}(f): \mathrm{CFK}^{-}\left(\Sigma^{1}, \alpha^{1}, \beta^{1}, w^{1}, z^{1}\right) \longrightarrow \mathrm{CFK}^{-}\left(\Sigma^{2}, \alpha^{2}, \beta^{2}, w^{2}, z^{2}\right) .
$$

Definition 6.5 Suppose that $F:(Y, K) \longrightarrow(Y, K)$ is a homeomorphism (fixing $K$ pointwise). Choose a Heegaard diagram $\left(\Sigma^{1}, \alpha^{1}, \beta^{1}, w^{1}, z^{1}\right)$ for $(Y, K, p)$. The map $F$ induces another homeomorphic diagram

$$
\left(\Sigma^{2}, \alpha^{2}, \beta^{2}, w^{2}, z^{2}\right)=\left(F\left(\Sigma^{1}\right), F\left(\alpha^{1}\right), F\left(\beta^{1}\right), F\left(w^{1}\right), F\left(z^{1}\right)\right),
$$

together with an induced homeomorphism

$$
f:\left(\Sigma^{1}, \alpha^{1}, \beta^{1}, w^{1}, z^{1}\right) \longrightarrow\left(\Sigma^{2}, \alpha^{2}, \beta^{2}, w^{2}, z^{2}\right) .
$$

We define the action of $F$ on the (projectivized) Floer homology of $(Y, K)$ to be the composite

$\operatorname{HFK}^{-}\left(\Sigma^{1}, \alpha^{1}, \beta^{1}, w^{1}, z^{1}\right) \stackrel{\Xi_{1,2}(f)_{*}}{\longrightarrow} \operatorname{HFK}^{-}\left(\Sigma^{2}, \alpha^{2}, \beta^{2}, w^{2}, z^{2}\right) \stackrel{\left(\Psi_{2,1}\right)_{*}}{\longrightarrow} \operatorname{HFK}^{-}\left(\Sigma^{1}, \alpha^{1}, \beta^{1}, w^{1}, z^{1}\right)$.

The resulting map will be denoted by

$$
A(F): \operatorname{HFK}^{-}(Y, K) / \pm 1 \longrightarrow \operatorname{HFK}^{-}(Y, K) / \pm 1 \text {. }
$$

Theorem 6.6 The above map

$$
F \in \operatorname{Diff}^{+}(Y, K) \mapsto A(F) \in \operatorname{Aut}\left(\operatorname{HFK}^{-}(Y, K) / \pm 1\right)
$$

descends to a well-defined action of the mapping class group of $(Y, K)$ on knot Floer homology.

Proof It is clear from the construction that if $F_{1}, F_{2} \in \operatorname{Diff}^{+}(Y, K)$, then $A\left(F_{1}\right) \circ A\left(F_{2}\right)=A\left(F_{1} \circ F_{2}\right)$.

Next, we claim that if $F_{t}:(Y, K) \longrightarrow(Y, K)$ is an isotopy with the property that $F_{0}$ is the identity map, then there is some $\epsilon>0$ with the property that for all $|t|<\epsilon, A\left(F_{t}\right)$ acts as the identity map. 
We argue as follows. Choose an allowed generic almost-complex structure, so that for any $\mathbf{x}, \mathbf{y} \in \mathbb{T}_{\alpha} \cap \mathbb{T}_{\beta}$, and any non-constant $\phi \in \pi_{2}(\mathbf{x}, \mathbf{y})$ with the property $\mu(\phi)=0$, the moduli space $\widehat{\mathfrak{M}}(\phi)$ is empty. It follows that in the continuation map $\Gamma$ (defined by counting time-dependent boundary conditions), if $t$ is sufficiently small and the moduli space for the continuation $\widehat{\mathfrak{M}}\left(\mathbf{x}, \mathbf{y}^{\prime}\right.$ ) (for $\mathbf{x} \in \mathbb{T}_{\alpha} \cap \mathbb{T}_{\beta}$ and $\left.\mathbf{y}^{\prime} \in \mathbb{T}_{\alpha}^{\prime} \cap \mathbb{T}_{\beta}^{\prime}\right)$ is non-empty, then the homotopy class $\phi$ must correspond to the constant map. It follows that $\Gamma: \mathrm{CFK}^{-}\left(\Sigma, \alpha^{1}, \beta^{1}, w, z\right) \longrightarrow$ $\mathrm{CFK}^{-}\left(\Sigma, \alpha^{2}, \beta^{2}, w, z\right)$ is the closest-point map, provided that $\alpha^{2}$ and $\beta^{2}$ are sufficiently close to $\alpha^{1}$ and $\beta^{1}$; and also that $\widehat{\mathfrak{M}}\left(\mathbf{x}^{\prime}, \mathbf{y}^{\prime}\right)$ is empty for all $\phi \in$ $\pi_{2}\left(\mathbf{x}^{\prime}, \mathbf{y}^{\prime}\right)$ with $\mu(\phi)=0$, where $\mathbf{x}^{\prime}, \mathbf{y}^{\prime} \in \mathbb{T}_{\alpha^{2}} \cap \mathbb{T}_{\beta^{2}}$.

Now, we choose $\alpha^{3}, \beta^{3}$ to be an exact Hamiltonian translate of the pair $\alpha^{2}, \beta^{2}$. We claim that in this case, for sufficiently small translates, the map induced by triangles is also a nearest point map. This is seen by identifying the map from the continuation map $\Gamma$ (associated to the isotopy of $\alpha^{3}$ with $\alpha^{2}$ and $\beta^{3}$ with $\beta^{2}$ ) with the map defined by counting pseudo-holomorphic triangles, and then appealing to the previous paragraph. The identification of $\Gamma$ and the triangle map (in a related context) can be found in [11, Proposition 11.3]; we sketch this argument here. The continuation map (in the case where only the $\beta$-circles are moving, while the $\alpha$-circles stay fixed, for notational simplicity) can be thought of as counting pseudo-holomorphic triangles with one corner smoothed out, which map one boundary edge to $\mathbb{T}_{\alpha}$, another to $\mathbb{T}_{\beta}$, the third to $\mathbb{T}_{\beta}^{\prime}$ (belonging to an isotopic translate of $\mathbb{T}_{\beta}$ ), and following some fixed isotopy of $\mathbb{T}_{\beta}$ to $\mathbb{T}_{\beta}^{\prime}$ along the rounded edge. Stretching out the rounded edge, we obtain a chain homotopy between this map, and the map induced by counting pseudoholomorphic triangles for the Heegaard triple $\left(\Sigma, \alpha, \beta, \beta^{\prime}, w, z\right)$, which is some cycle in $\mathrm{CFK}^{-}\left(\Sigma, \beta, \beta^{\prime}, w, z\right)$ (which we can think of as the relative invariant of the isotopy). The fact that both maps induce Maslov grading-preserving isomorphisms on Floer homology ensures that the relative invariant represents the top-dimensional homology generator of $\operatorname{HFK}^{-}\left(\Sigma, \beta, \beta^{\prime}, w, z\right)$. This completes the identification of the continuation map with the map induced by pseudoholomorphic triangles, on the level of homology. (We have explained here the case where only the $\beta$ circles are moving; the case where both $\alpha$ - and $\beta$-circles are moving follows with straightforward notational changes.)

Finally, observe that the map induced by the homeomorphism $F_{t}$ is also a closest-point map. Thus, for all $t$ sufficiently small, $A\left(F_{t}\right)$ acts by the identity on homology.

By the compactness of $[0,1]$ we conclude that any $F$ which is isotopic to the identity acts by the identity map on knot Floer homology. Consequently 
the action of $\operatorname{Diff}^{+}(Y, K)$ descends to an action of $\operatorname{Diff}^{+}(Y, K) / \operatorname{Diff}_{0}^{+}(Y, K)=$ $\operatorname{MCG}(Y, K)$ on $\operatorname{HFK}^{-}(Y, K)$, concluding the proof.

Note that if $F:\left(Y_{1}, K_{1}, p_{1}\right) \longrightarrow\left(Y_{2}, K_{2}, p_{2}\right)$ is a homeomorphism, then Definition 6.5 can be adapted to define a map

$$
\operatorname{HFK}^{-}\left(Y_{1}, K_{1}, p_{1}\right) \longrightarrow \operatorname{HFK}^{-}\left(Y_{2}, K_{2}, p_{2}\right),
$$

which is well-defined up to the above action of the mapping class group of $\left(Y_{2}, K_{2}\right)$.

\section{References}

[1] S. Akbulut and B. Ozbagci. Lefschetz fibrations on compact Stein surfaces. Geom. Topol., 5:319-334 (electronic), 2001.

[2] F. Arikan. On the support genus of a contact structure. J. Gökova Geom. Topol. GGT, 1:92-115, 2007.

[3] J. S. Birman and W. M. Menasco. Stabilization in the braid groups. II. Transversal simplicity of knots. Geom. Topol., 10:1425-1452 (electronic), 2006.

[4] Y. Chekanov. Differential algebra of Legendrian links. Invent. Math., 150(3):441-483, 2002.

[5] J. Epstein, D. Fuchs, and M. Meyer. Chekanov-Eliashberg invariants and transverse approximations of Legendrian knots. Pacific J. Math., 201(1):89-106, 2001.

[6] J. Etnyre, L. Ng, and V. Vértesi. Legendrian and transverse twist knots. arXiv:1002.2400, 2010.

[7] J. B. Etnyre and K. Honda. Cabling and transverse simplicity. Ann. of Math. (2), 162(3):1305-1333, 2005.

[8] A. Hatcher and W. Thurston. Incompressible surfaces in 2-bridge knot complements. Invent. Math., 79:225-246, 1985.

[9] K. Honda, W. H. Kazez, and G. Matić. The contact invariant in sutured Floer homology. Invent. Math., 176(3):637-676, 2009.

[10] K. Honda, W. H. Kazez, and G. Matić. On the contact class in Heegaard Floer homology. J. Differential Geom., 83:289-311, 2009.

[11] R. Lipshitz. A cylindrical reformulation of Heegaard Floer homology. Geom. Topol., 10:955-1097 (electronic), 2006.

[12] P. Lisca, P. S. Ozsváth, A. I. Stipsicz, and Z. Szabó. Heegaard Floer invariants of Legendrian knots in contact three-manifolds. J. Eur. Math. Soc. (JEMS), 11(6):1307-1363, 2009. 
[13] C. Manolescu, P. S. Ozsváth, and S. Sarkar. A combinatorial description of knot Floer homology. Ann. of Math., 169:633-660, 2009.

[14] C. Manolescu, P. S. Ozsváth, Z. Szabó, and D. Thurston. On combinatorial link Floer homology. Geom. Topol., 11:2339-2412, 2007.

[15] L. Ng, P. S. Ozsváth, and D. P. Thurston. Transverse knots distinguished by knot Floer homology. J. Symplectic Geom., 6:461-490, 2008.

[16] L. Ng and L. Traynor. Legendrian solid-torus links. J. Symplectic Geom., 2:411-443, 2004.

[17] Y. Ni. Knot Floer homology detects the Thurston norm. Invent. Math., 170:577$608,2007$.

[18] P. Ozsváth and Z. Szabó. Heegaard Floer homology and alternating knots. Geom. Topol., 7:225-254 (electronic), 2003.

[19] P. Ozsváth, Z. Szabó, and D. Thurston. Legendrian knots, transverse knots and combinatorial Floer homology. Geom. Topol., 12:941-980, 2008.

[20] P. S. Ozsváth and Z. Szabó. Absolutely graded Floer homologies and intersection forms for four-manifolds with boundary. Adv. Math., 173:179-261, 2003.

[21] P. S. Ozsváth and Z. Szabó. Holomorphic disks and knot invariants. Adv. Math., 186(1):58-116, 2004.

[22] P. S. Ozsváth and Z. Szabó. Holomorphic disks and three-manifold invariants: properties and applications. Ann. of Math. (2), 159(3):1159-1245, 2004.

[23] P. S. Ozsváth and Z. Szabó. Holomorphic disks and topological invariants for closed three-manifolds. Ann. of Math. (2), 159(3):1027-1158, 2004.

[24] P. S. Ozsváth and Z. Szabó. Heegaard Floer homology and contact structures. Duke Math. J., 129(1):39-61, 2005.

[25] P. S. Ozsváth and Z. Szabó. Holomorphic triangles and invariants for smooth four-manifolds. Adv. Math., 202(2):326-400, 2006.

[26] J. A. Rasmussen. Floer homology of surgeries on two-bridge knots. Algebr. Geom. Topol., 2:757-789 (electronic), 2002.

[27] J. A. Rasmussen. Floer homology and knot complements. PhD thesis, Harvard University, 2003.

[28] A. Reid and G. Walsh. Commesurability classes of 2-bridge knot complements. Algebr. Geom. Topol., 8:1031-1057, 2008.

[29] B. Sahamie. Dehn twists in Heegaard Floer homology. Alg. Geom. Top., 10:465$524,2010$.

[30] V. Vértesi. Transversely non simple knots. Algebr. Geom. Topol., 8:1481-1498, 2008. 